\title{
Exosomes Derived From miR-214-3p \\ Overexpressing Mesenchymal Stem Cells Promote Myocardial Repair
}

\section{Wenwu Zhu}

Xuzhou Central Hospital

\section{Qingjie Wang}

The affilitated Changzhou NO.2 hospital of Nanjing medical university

Jian Zhang

The first Affiliated hospital of Nanjing Medical university

\section{Ling Sun}

Nanjing Medical University Affiliated Changhzou No.2 People's Hospital

Wei Du

Xuzhou Central Hospital

\section{Yujie Wu}

The First Affiliated Hospital of Nanjing Medical University

\section{Yue Cao}

Xuzhou Central Hospital

\section{Boyu Chi}

Changzhou second People's Hospital

Jing Li

Xuzhou Central Hospital

\section{Yueyue Feng}

Xuzhou Central Hospital

\section{Ailin Zou}

Changzhou Second People's Hospital

\section{Lipeng Mao}

Changzhou City Second People's Hospital

\section{Jianguang Jiang}

Changzhou City Second People's Hospital

\section{Yuan Ji}

Changzhou City Second People's Hospital

\section{Bing Han ( $\sim$ hbing777@hotmail.com )}

Xuzhou Central Hospital https://orcid.org/0000-0002-3523-0573 


\section{Research Article}

Keywords: mesenchymal stem cells (MSCs), acute myocardial infarction (AMI), miR-214, miR-2140E-Exo Posted Date: July 26th, 2021

DOl: https://doi.org/10.21203/rs.3.rs-721088/v1

License: (c) (i) This work is licensed under a Creative Commons Attribution 4.0 International License. Read Full License 


\section{Abstract}

Aims Exosomes are known as nanovesicles that are naturally secreted冈playing an essential role in stemmediated cardioprotection. This study mainly focused on investigating if exosomes derived from miR214 overexpressing mesenchymal stem cells (MSCs) show more valid cardioprotective ability in a rat model of acute myocardial infarction (AMI) and its potential mechanisms.

Methods Exosomes were isolated from control MSCs (Ctrl-Exo) and miR-214 overexpressing MSCs (miR$214^{\mathrm{OE}}$-Exo) and then they were delivered to cardiomyocytes and endothelial cells in vitro under hypoxia and serum deprivation (H/SD) condition or in vivo in an acutely infarcted Sprague-Dawley rat heart. Regulated genes and signal pathways by miR-214 ${ }^{\mathrm{OE}}$-Exo treatment were explored using western blot analysis and luciferase assay.

Results In vitro, miR-214 ${ }^{\mathrm{OE}}$-Exo enhanced migration, tube-like formation in endothelial cells. In addition, miR-214 ${ }^{\mathrm{OE}}$-Exo ameliorated the survival of cardiomyocytes under H/SD. In the rat AMI model, compared to Ctrl-Exo, miR-214 ${ }^{\mathrm{OE}}$-Exo reduced myocardial apoptosis, and therefore reduced infarct size and improved cardiac function. Besides, miR-214 ${ }^{\mathrm{OE}}$-Exo accelerated angiogenesis in peri-infarct region. Mechanistically, we identified that exosomal miR-214-3p promoted cardiac repair via targeting PTEN and activating p-AKT signal pathway.

Conclusion Exosomes derived from miR-214 overexpressing MSCs have greatly strengthened the therapeutic efficacy for treatment of AMI by promoting cardiomyocyte survival and endothelial cell function.

\section{Introduction}

Acute myocardial infarction (AMI) irreversibly causes cardiomyocytes death and myocardial remodeling, leading to ischemic heart disease ${ }^{1}$. At present, surgical intervention and thrombolysis are important treatment methods, which may induce reperfusion injuries during and after surgical procedures, such as myocardial cell death and microvascular injury ${ }^{2,3}$. It is necessary to develop effective complementary therapies to reduce the loss of cardiomyocytes.

In recent years, microRNAs (miRNAs) have been applied to treatment cardiovascular diseases ${ }^{4-7}$. MiRNAs controls gene-expressing by matching with 3 'untranslated region (UTR) of targeted mRNA, leading to mRNA degradation or translation inhibition ${ }^{8}$. More and more studies have shown that miRNAs take part in the regulation of myocardial angiogenesis, fibrosis and apoptosis after myocardial infarction, and become potential drug candidates for the treatment of myocardial infarction ${ }^{9-11}$. However, the poor stability of miRNAs in vivo and poor cellular uptake limit their clinical application ${ }^{12}$. Several methods have been developed for miRNAs delivery, including liposomes, viral vectors, hydrodynamic injection and nanocarriers, but these methods are related to carrier toxicity, low delivery efficiency and adverse immune reactions. Therefore, there is a great need for reliable and efficient delivery methods. 
Exosomes are a group of cell-secreted heterostructures, ranging in diameter from 30 to $150 \mathrm{~nm}^{13}$. The lipid membrane structure of exosomes is similar to that of liposomes. Thus, exosome can protect nucleic acids from degradation, and facilitate transport of miRNAs, mRNAs, and proteins to receptor cells with no virulence and adverse immunogenicity ${ }^{14-16}$. Genetic engineering of miRNA in exosomes can further enhance the protective effects on different diseases ${ }^{17,18}$. Therefore, exosomes may be a better alternative for the miRNA delivery in vivo. It is reported that exosomes from cardiac progenitor cells (CPCs), mesenchymal stem cells (MSCs), induced pluripotent stem cells and embryonic stem cells exert protective effects during cardiac repair as a cell-free therapy ${ }^{19-21}$.

MiRNA-214 mediates anti-apoptosis and therapeutic angiogenesis. Lv et al. reported that miRNA-214 inhibited apoptosis of infarcted cardiomyocytes via PTEN/Akt by targeting PTEN, and promoted endothelial cell migration and angiogenesis by targeting ATM ${ }^{22,23}$. In this study, we first isolated miRNA214-rich exosomes (miR-214 ${ }^{\mathrm{OE}}$-Exo) from genetically modified mesenchymal stem cells, and then delivered these miR-214-exosomes to the heart tissue via intra myocardial injection in order to promote cardiac function recovery. The exosome-mediated specific miRNAs delivery might have the possible effect on the treatment of AMI.

\section{Methods}

\section{Ethical statement}

Animal experiments were conducted according to the Guidelines for the Care and Use of Laboratory Animals and were approved by Ethics Committee of Xuzhou Central Hospital. Surgical operations are performed under anesthesia by sodium pentobarbital $(50 \mathrm{mg} / \mathrm{kg})$ injecting intraperitoneally.

\section{Cell culture and identification}

Human umbilical mesenchymal stem cells (HuMSCs) were gained from Clinical Center of Reproductive Medicine in Nanjing. HuMSCs were cultured with Eagle's Minimum Essential Medium (EMEM, Gibco, USA) containing $10 \%$ fetal bovine serum(FBS, Gibco, USA), $5 \mathrm{ng} / \mathrm{ml}$ epidermal growth factor and $5 \mathrm{ng} / \mathrm{ml}$ alkaline fibroblast growth factor. Human umbilical vein endothelial cells were cultured in Dulbecco modified eagle medium (DMEM, Gibco, USA) containing fetal bovine serum (10\%), $100 \mu \mathrm{g} / \mathrm{ml}$ streptomycin, $100 \mathrm{U} / \mathrm{ml}$ penicillin, and $110 \mathrm{mg} / \mathrm{ml}$ sodium acetone. All those cells were cultured in an incubator which contains $5 \% \mathrm{CO}_{2}$ at $37^{\circ} \mathrm{C}$. HuMSCs used for experiments were between passages 3 and 6. Oil red staining or alcian blue were used to examine differentiation capacity of HuMSCs for adipogenesis and chondrogenesis.

\section{Neonatal rat cardiomyocytes (NRCMs) isolation and culture}

Myocardial cells were extracted from those 1-3-days old suckling rats. Ventricles were cleaned and shredded using $1 \times$ ADS ( $\mathrm{NaCl} 68 \mathrm{~g} / \mathrm{L}$, HEPES 47.6g/L, Na2HPO4 1.38g/L, Glucose 6g/L, KCl 4g/L and MgSO4 1g/L) buffer solution with pH 7.35-7.45. After that, tissues were digested by $0.6 \mathrm{mg} / \mathrm{ml}$ 
pancreatin and $0.4 \mathrm{mg} / \mathrm{ml}$ collagenase II. Collect the supernatant and add one-fifth horse serum of the volume of supernatant after each digestion. Finally, the cells were purified and cultured in DMEM consisting of $10 \%$ horse serum, $5 \%$ fetal bovine serum, $100 \mu \mathrm{g} / \mathrm{ml}$ streptomycin and $100 \mathrm{U} / \mathrm{ml}$ penicillin, and placed in an incubator with a $\mathrm{CO}_{2}$ content of $5 \%$ and a temperature of $37^{\circ} \mathrm{C}$.

\section{Extraction and identification of exosomes}

Isolation of exosome was proceed as described previously ${ }^{9}$. Briefly, huMSCs with $80 \%$ confluence were washed with PBS and then cocultured in exosome free medium for $48 \mathrm{~h}$. The supernatant were centrifuged at $1500 \mathrm{~g}$ for 30 minutes and incubated with Exosome Isolation Reagent (C10130-2, RiboBio, China) for 12 hours at a temperature of $4^{\circ} \mathrm{C}$, and centrifuged at $2000 \mathrm{~g}$ for half an hour. The precipitates were suspended with PBS and stored at $80^{\circ} \mathrm{C}$. Bicinchoninic acid assay (BCA, Thermo Fisher Scientific, Waltham, MA) was used to detect exosomes protein concentration by measuring absorbance at $562 \mathrm{~nm}$. Transmission electron microscopy (TEM; Hitachi H-7650; Japan) was used to observe morphology of exosomes and western blot was carried out to detect surface markers CD81, CD63 and TSG101. Nanoparticle Tracking Analysis (NTA) was used to analyze the size and concentration of exosomes.

\section{Lentiviral constructions and infection}

The lentivirus was gained from Shanghai Genechem Co., LTD. There are two types of lentiviral recombinant vectors. One is Ubi-MCS-SV40-EGFP-IRES-puromycin, used as hsa-miR-214-3p OE (overexpression of hsa-miR-214-3p) virus. Another is H1-MCS-CMV-EGFP, used as hsa-miR-214-3p NC (Control) virus. HuMSCs were incubated in 24 -well plates, grow to $50 \%$ confluence, and infected with hsamiR-214-3p ${ }^{O E}$ virus or hsa-miR-214-3p ${ }^{N C}$ virus, respectively. The fluorescence signals were observed and the expression of hsa-miR-214-3p was detected by qRT-PCR.

\section{Exosome uptake assay}

Exosomes were labelled by $1 \mu \mathrm{mol}$ Dil (ThermoFisher, USA) and were cultured with cells for 6 or $24 \mathrm{~h}$ in vitro. Nuclei were stained with 4',6-diamidino-2-phenylindole (DAPI, Beyotime, Shanghai, China). Zeiss laser-scanning confocal microscope (LSM5 Live, Carl Zeiss, Germany) were used to evaluate the absorbance of exosomes.

\section{Migration assay}

HUVECs were planted on 6-well plate with $2 \times 10^{5}$ cells/well. Cells were treated with $1 \mathrm{~mL}$ test medium for 24 hours. After that, cells were rinsed and scratched by P200 pipette tip. Cell migration was evaluated 24 hours later. All samples were observed with three replicates.

\section{Tube formation of HUVECs assay}

Capillary tube formation assay was used to observe angiogenesis of HUVECs. HUVECs were incubated in 96-well plate coated with growth factor reduced Matrigel (356,230; BD Biosciences, SanJose, CA, USA). 
Six hours later, formation of capillary-like tubes was observed and the tube length was analyzed by Image $\mathrm{J}$ software. All experiments were carried out with three replicates.

\section{Immunofluorescence and TdT-mediated dUTP nick-end labeling staining}

Cardiomyocytes were fixed with $4 \%$ paraformaldehyde for $15 \mathrm{~min}$, and then permeabilized with $0.5 \%$ Triton X-100 for 15 min. After that, cells were incubated with 10\% goat serum for 2 hours, a-actinin primary antibody (1:150; A7811; Sigma, Santa Clara, CA) overnight at $4^{\circ} \mathrm{C}$ and second antibody for 1 hour at room temperature. TdT-mediated dUTP nick-end labeling (TUNEL) staining was used to examine apoptotic cells following manufacturer's instructions (Roche, Mannheim, Germany).

\section{MI model establishing and exosomes injection}

Sprague Dawley rats (Male, 6-8 weeks) were provided by animal center of Nanjing Medical University (Nanjing, China). Rats were injected $50 \mathrm{mg} / \mathrm{kg}$ sodium pentobarbital and mechanical ventilated via orotracheal intubation. The left anterior descending coronary artery was ligated $1.5 \mathrm{~mm}$ at the lower edge of the left auricle by thoracotomy. Exosomes (50ug) were injected into 4 portions at the border of the infarction area. All surgeries and analyses were blind interventions.

\section{Cardiac function assessment}

The cardiac function was measured by transthoracic echocardiography 28 days after exosome injection with VEVO 2000 high-resolution micro-imaging system. A $30 \mathrm{MHz}$ transducer was used to measure the left ventricular short-axis in M-mode. The left ventricular ejection fraction (LVEF) and left ventricular shortening rate (LVFS) were calculated by Vevo2000 workstation software.

\section{Masson trichrome staining and Hematoxylin-Eosin staining}

Heart tissues were collected, fixed and sliced to $5 \mu \mathrm{m}$. Mason's trichrome staining as described above ${ }^{9}$. The proportion of infarct area is the sum of infarct area of each section/sum of LV area of each section $x$ $100 \%$. Hematoxylin-Eosin (HE) stain was used to measure the level of inflammatory cells.

\section{Fluorescence in situ hybridization (FISH) of miR-214}

MiR-214 ${ }^{\mathrm{OE}}$ exosomes (50ug) were injected at the edge of the infarction area. After 6 hours, the rats were sacrificed. The miR-214 oligonucleotide probes (Sangon Biotech, China, $8 \mathrm{ng} / \mu \mathrm{L}$ ) was incubated with Cy3 or FAM in pre-hybridization buffer at $37^{\circ} \mathrm{C}$ for $1 \mathrm{~h}$, and then hybridized overnight at $37^{\circ} \mathrm{C}$. With sections 2 $\times$ SSC, $1 \times$ SSC, $0.5 \times$ SSC rinsing and DAPI staining, the internalization of miR-214 in recipient cells or myocardium was observed by confocal microscope.

\section{Immunofluorescence}

Immunofluorescence was performed as reported previously ${ }^{24}$. Briefly, heart tissue were collected and then fixed, embedded, and sectioned. After that, the section was stained with primary antibody anti-actin and 
CD31 (1:200, ab7388, Abcam, Cambridge). The nuclei was stained with DAPI.

\section{Western blot analysis}

Western blotting was performed with a standard protocol as previously described ${ }^{9}$. Antibodies were used as follows: GAPDH (1:1000; 5174S; Cell Signaling Technology), Bcl-2 (1:1000; 2870S; Cell Signaling Technology), AKT (1:1000; 4961S; Cell Signaling Technology), p-AKT (1:1000; 4060S; Cell Signaling Technology), BAX (1:1000; 5023, Cell Signaling Technology), PTEN (1:1000; ab31392, Abcam).

\section{Quantitative real-time polymerase chain reaction analysis}

Total RNAs were extracted by Trizol and treated with RNase-free DNase I ( $1 / 20 \mu \mathrm{L}$, Promega Corp, Madison, WI). Reverse transcription of cDNAs was performed using Reverse Transcription Kit (Takara, Dalian, China). Polymerase chain reaction analysis (PCR) analysis was performed with SYBR green PCR Master Mix (Applied Biosystems, Foster, CA, USA) on ABI-7900 Real-Time PCR Detection System (7900HT; Applied Biosystems). The exosomal level of miR-214-3p was normalized to that of cel-miR-39 (C39). The cellular miR-214-3p expression was normalized to U6. The cellular expression of PTEN mRNA was normalized to that of glyceraldehyde3-phosphate dehydrogenase (GAPDH). The related gene expression was normalized to that of GAPDH. The primer sequences are listed in Additional file: Table S1.

\section{Flow cytometry analysis}

Apoptosis of HUVECs were examined by flow cytometry using Annexin V Alexa Fluor647/PI/Apoptosis detection kit (Fcmacs Biotech, Nanjing, China). Briefly, cells were washed, digested and resuspended in a binding buffer with double distilled water at a ratio of 1:3, incubated by $5 \mu$ Innexin $V$ and $10 \mu \mathrm{l}$ propidium iodide (PI) for 15 min at room temperature in the dark. After that, $400 \mu \mathrm{lPBS}$ was added and flow cytometry analysis was performed on FAC Scan. Data were analyzed by FlowJo software.

\section{Luciferase assay}

The PTEN 3'UTR cDNA sequences containing mir-214-3p binding site was inserted into pmir-GLOpromoter vector (Promega, Madison, USA). HEK-293T cells were transfected with miR-NC or miR-214-3p mimics and seeded into 96-well plates. The sequences of miRNAs transfected were shown in Additional file: Table S2. After that, cells were co-transfected with $100 \mathrm{ng}$ pmiR-GLO-PTEN-WT or pmiR-GLO-PTENMUT. The luciferase activity was detected by EnSpire ${ }^{\circledR} 2300$ Multimode Plate Reader (Perkin Elmer Singapore Pte. Ltd., Singapore) 24 hours after transfection. luciferase activities of firefly were normalized to Renilla luciferase activity.

\section{Statistical Analysis}

All data are expressed in the way of meantstandard deviation. The two-tailed test was used to compare the means between the two groups, and one-way analysis of variance was used for multiple experimental 
groups. SPSS statistical software (version 17.0, SPSS Inc., Chicago, IL) was used for statistical analysis. $P$ value of less than 0.05 was considered statistically significant.

\section{Results}

\section{Characterization of huMSCs and exosomes derived from huMSCs}

The morphology of huMSCs was shown in Figure 1a by a light microscope. Oil red staining and alcian blue staining confirmed multiple differentiation potential of huMSCs for adipogenesis and chondrogenesis. Lentiviral modifications of miR-214-MSCs (huMSCs transfected with lentiviruses containing miR-214) and Ctrl-MSCs (huMSCs transfected with lentiviruses containing miR-214 negative control) were confirmed by examining green fluorescence using fluorescent microscopy (Figure 1c). Compared with the Ctrl-MSC group, the cellular and exosomal miR-214-3p expression in miR-214-MSC group were increased (Figure 1d). Transmission electron microscopy scanning showed the morphology of both Ctrl-Exo and miR-214 ${ }^{\mathrm{OE}}$-Exo were round or disk-shaped. (Figure 1e). Then, we analyzed the diameters of the Ctrl-Exo and miR-214 ${ }^{\mathrm{OE}}$-Exo using NanoSight. Most Ctrl-Exo and miR-214 ${ }^{\mathrm{OE}}$-Exo had a size of about 30-150 nm (Figure 1f-g). Exosome specific markers of TSG101, CD81 and CD63 were positive in Ctrl-Exo and miR-214 ${ }^{\mathrm{OE}}$-Exo, Calnexin were not detected among exosome proteins. Dil labelled exosomes were co-cultured with HUVECs and NRCMs for $6 \mathrm{~h}$ and $24 \mathrm{~h}$ under H/SD. Confocal images confirmed the uptake of labeled exosomes by NRCMs and HUVECs in a time dependent manner.

\section{miR-214 ${ }^{\mathrm{OE}}$-Exo conferred better protective effects on HUVECs and NRCMs than Ctrl-Exo under H/SD in vitro}

Exosomes from stem cell exerts therapeutic benefits via promoting neovascularization. Hence, effects of miR-214 ${ }^{\mathrm{OE}}$-Exo on migration, tube formation, and anti-apoptosis in HUVECs and NRCMs were evaluated. Compared with PBS and Ctrl-Exo groups, migration rate of HUVECs was markedly improved in miR-214 ${ }^{\mathrm{OE}}$. Exo group (Figure 2a). Tube formation of HUVECs also significantly increased in miR-214 ${ }^{\mathrm{OE}}$-Exo compared with PBS and Ctrl-Exo groups (Figure 2b). TUNEL showed reduced apoptosis of NRCMs in the miR-214 ${ }^{\mathrm{OE}}$-Exo group compared with PBS and Ctrl -Exo groups. Taken together, these results showed that miR-214 ${ }^{\mathrm{OE}}$-Exo had cell protection effects on HUVECs and NRCMs under H/SD in vitro.

\section{miR-214 ${ }^{\mathrm{OE}}-$ Exo effectively preserved cardiac function in a rat $\mathrm{MI}$ model}

MiR-214 ${ }^{\mathrm{OE}}$-Exo, Ctrl -Exo, or PBS (AMI control group) were injected into the border area of infarcted hearts (Figure 3a). The distribution of Dil-labeled miR-214 ${ }^{\mathrm{OE}}$-exo (Dil-Exosome) in the infarct heart was examined $6 \mathrm{~h}$ after injury. As is shown in Figure 3, there were large Dil-labeled positive areas near exothelium (Figure $3 b$ ) and in the myocardium (Figure 3c), indicating that miR-214 ${ }^{\mathrm{OE}}$-Exo were effectively presented in myocardial cells and endothelial cells in the infarcted hearts. Moreover, FISH showed that miR-214 was concentrated $6 \mathrm{~h}$ after injection, mainly in the peri-nuclear region of the myocardium (Figure 3d). At 4 weeks after AMI, compared with the PBS group and the Ctrl-Exo group, LVEF and LVFS were significantly 
improved in the miR-214 ${ }^{\mathrm{OE}}$-Exo group (Figure 4a). Masson staining showed that fibrosis area of mir$214^{\mathrm{OE}}$-Exo group was significantly reduced compared with PBS and Ctrl exo groups (Figure 4b). Compared with the Ctrl -Exo and AMI groups, the miR-214 ${ }^{\mathrm{OE}}$-Exo group also had significantly less inflammatory cell infiltration (Figure 4c). These results indicated that miR-214 ${ }^{\mathrm{OE}}$-Exo played a potential role in the maintenance of cardiac function in infarcted rats.

\section{miR-214 ${ }^{\mathrm{OE}}$-Exo promoted angiogenesis and cardiomyocyte survival in $\mathrm{MI}$ hearts}

In order to reveal the mechanism of improved cardiac function after in vitro exosome treatment, immunofluorescence staining of capillaries and arterioles were performed using CD31 and a-SMA antibodies. The results showed that the capillary density of miR-214 ${ }^{\mathrm{OE}}$-Exo group was significantly higher than that of Ctrl -Exo and AMl groups at 4 weeks after infarction (Figure $5 \mathrm{a}$ ). Figure $5 \mathrm{~b}$ showed the same trends in capillary density and arteriole density. Figure $5 \mathrm{c}$ and $5 \mathrm{~d}$ showed the TUNEL and Cleaved Caspase-3 staining of 4-week infarcted rat hearts to revel the anti-apoptotic effect of exosomes. Compared with PBS, the number of apoptotic cells in the border zone of infarcted myocardium treated with exosomes decreased significantly. Moreover, TUNEL+ and Cleaved Caspase- $3+$ cells were minimal in the miR-214 ${ }^{\mathrm{OE}}$-Exo group. These results suggest that miR-214 ${ }^{\mathrm{OE}}$-Exo enhances angiogenesis and cell survival, which in turn improved cardiac repair ability.

\section{Exosomal miR-214-3p attenuated cardiomyocyte injury via regulation of p-AKT by targeting PTEN}

Emerging evidence has revealed that the AKT signaling pathway plays an important role in regulating cell survival. Expression of p-AKT was tested to elucidate if exosomal miR-214-3p promoted cardiomyocyte survival via AKT signaling pathway. Expression of p-AKT in NRCMs was greatly diminished under H/SD condition, whereas miR-214 ${ }^{\mathrm{OE}}$-Exo increased $\mathrm{p}$-Akt expression under $\mathrm{H} / \mathrm{SD}$ condition. Bcl2/Bax protein level was significantly higher compared with the Ctrl -Exo and AMI groups (Figure 6a). AKT inhibitor (MK2206) was used to further validate the relationship between AKT and miR-214 ${ }^{\mathrm{OE}}$-Exo in the regulation of cardiomyocyte survival. MK2206 significantly inhibited p-AKT induced by miR-214 ${ }^{\mathrm{OE}}$-Exo. In addition, MK2206 reversed miR-214 ${ }^{\mathrm{OE}}$-Exo induced BCL2/Bax protein levels. In order to further explore the mechanism of miR-214 ${ }^{\mathrm{OE}}$-Exo in promoting angiogenesis and anti-apoptosis, potential target genes of miR-214-3p in endothelial cells and cardiomyocytes were predicted based on the online repository of miRNA targets. We confirmed that PTEN may be a potential target for neovascularization and cardiomyocyte survival, and investigated the relationship between exosome miR-214-3p and PTEN. Figure 6e showed wild-type PTEN (Wt-PTEN) and mutant-type PTEN (Mut-PTEN) luciferase reporter gene vector. Wt-PTEN or Mut-PTEN was transfected into HEK293T cells with miR-214-3p mimics or miR-214-3p mimics NC. The transfection efficiency of the mimics and mimics NC was verified by qRT-PCR (Additional file: Figure S1). Using Western blotting analysis, decreased expressional levels of PTEN were detected in HUVECs and NCRMs after administration of the miR-214 ${ }^{\mathrm{OE}}$-Exo (Figure $6 \mathrm{f}-\mathrm{g}$ ). The relative luciferase activity decreased when miR-214-3p mimics were co-transfected with Wt-PTEN, but not with the Mut- 
PTEN (Figure 6h). In summary, we confirmed that miR-214-3p negatively modulates PTEN by directly targeting its 3 '-UTR.

\section{miR-214 ${ }^{\mathrm{OE}}$-Exo promote migration, tube formation and apoptosis inhibition by targeting PTEN}

In vitro rescue tests were processed to investigate the association of miR-214 ${ }^{\mathrm{OE}}$-Exo and PTEN. PTEN and vector were inserted into HUVECs by transfection and analyzed by qRT-PCR (Figure 7a). PTEN expression was also detected in HUVECs treated with miR-214 ${ }^{\mathrm{OE}}$-Exo. Results demonstrated that HUVECs transfected with Vector showed a significantly lower expression of PTEN when compared to PTEN after administration of miR-214 ${ }^{\mathrm{OE}}$-Exo (Figure $7 \mathrm{~b}$ ). In tube formation experiments, we observed that overexpression of PTEN inhibited angiogenesis during co-administration with miR-214 ${ }^{\mathrm{OE}}$-Exo (Figure 7cd). In addition, scratch tests showed that overexpression of PTEN eliminated the positive effects of miR$214^{\mathrm{OE}}$-Exo on HUVECs migration (Figure 7e-f). Annexin V-FITC/PI flow cytometry demonstrated that the expression of PTEN could effectively reverse the protective effect of anti-apoptosis induced by administration of miR-214 ${ }^{\mathrm{OE}}$-Exo (Figure $7 \mathrm{~g}-\mathrm{h}$ ). We also found that incubation of miR-214 ${ }^{\mathrm{OE}}$-Exo and PTEN could significantly lessen the effects of miR-214 ${ }^{\mathrm{OE}}$-Exo on anti-apoptosis in NRCMs compared with miR-214 ${ }^{\mathrm{OE}}$-Exo and Vector(Figure 7i-j). Therefore, we concluded that exosomal miR-214-3p enhanced angiogenesis, migration, and apoptosis repression by targeting PTEN.

\section{Discussion}

The results of this study showed that miR-214 ${ }^{\mathrm{OE}}$-Exo promoted the migration, inhibited apoptosis, and improved tubular structure formation under H/SD in HUVECs or NRCMs. Furthermore, the results showed that miR-214 ${ }^{\mathrm{OE}}$-Exo was superior to Ctrl-Exo in protecting cardiac function and inhibiting fibrosis. The protective effects of miR-214 ${ }^{\mathrm{OE}}$-exo were at least partially mediated through the Akt signaling pathway.

MiRNAs have been proved to be effective therapeutic agent for $\mathrm{AMI}^{4}$. The effective and safe delivery system is a key to miRNA-based therapy ${ }^{25}$. Both viral and non-viral delivery systems have been used for target gene suppression. The advantage of viral vectors is high delivery efficiency. However, the toxicity, immunogenicity and tumorigenicity of viral vectors limit clinical application of viral vectors in vivo $^{26,27}$. Non-viral approaches seem more promising than viral vectors. However, endocytosis during liposome transfection cause endosome rupture or leakage, and trigger adverse inflammation and apoptosis ${ }^{28}$. Recently, studies shows that exosomes are novel RNA delivery tools without endosomal escape $^{29,30}$. Moreover, exosomes can enter deep tissues when internalized into the multivesicular body (MVB) of the recipient cell, which is then released again and internalized into the MVB of the secondary recipient cell ${ }^{31,32}$. In conclusion, exosomes have good biocompatibility, high cell uptake efficiency and biological safety, and can be used as an ideal vehicle for miRNAs delivery ${ }^{14,33}$. For example, MIF-Exo protect heart function by promoting angiogenesis, inhibiting apoptosis, reducing fibrosis by upregulating 
miR-133A-3p $p^{9}$. Sun et al. reported that hypoxic MSC-derived exosome exert cardioprotective effects via the exosomal IncRNAUCA1/miR873-5p/XIAP axis ${ }^{10}$.

Gene modification to produce exosomes is cheap, convenient and efficient, compared with electroporation and ultrasound methods. Exosomes derived from genetically modified stem cells have been used effectively to repair injured hearts as a new cell-free therapy ${ }^{34-36}$. Ma et al. reported that exosomes from AKT modified MSCs can promote angiogenesis and cardiac regeneration ${ }^{37}$. Sun et al. reported that HIF-1a overexpression in MSC-derived exosomes promotes heart repair by enhanced angiogenesis ${ }^{37}$. Our previous studies also showed that MIF engineered MSC-derived exosomes can reduce myocardial infarction size and improve cardiac function ${ }^{9}$. However, further studies of exosomes therapy are needed to ensure safety and effectiveness.

Our data show the anti-apoptotic and pro-angiogenic effects of exosomes released by miR-214 ${ }^{\mathrm{OE}}-\mathrm{MSC}$ via phosphatase and tensin homolog / Akt signal transduction. The exosome regulates the intracellular signaling pathway, in which exosome miRNAs play important roles in many biological processes. It's reported that miR-214-3p protected $\mathrm{H} 2 \mathrm{O} 2$ induced cardiomyocytes apoptosis ${ }^{22}$. The deletion of miR-214 gene aggravates cell death induced by ischemia-reperfusion and leads to deterioration of cardiac function. The miR-214 gene knockout led to more severe cell death and decreased left ventricular function, whereas miR-214 is upregulated in ischemic heart and is related to severity of the disease process $^{38-40}$. MiR-214 was reported to be secreted by exosomes of human endothelial cells ${ }^{23}$. This study confirmed that miR-214 modified huMSCs can reduce the area of myocardial fibrosis and improve cardiac function in ischemic hearts. MiR-214 was reported to regulate PTEN in H9C2 cardiomyocytes. Our study also found that upregulation of miR-214-3p in exosomes enhanced Akt phosphorylation via inhibiting PTEN in the recipient cells, while this effect can be eliminated by overexpression of PTEN. Taken together, our study confirmed the protective effects of miR-214-3p enriched exosomes in post-MI repair.

There are some limitations in current study. Firstly, intramyocardial injection was used to deliver exosomes to target sites. However, this could cause injury of heart and leakage of injected miR-214 $\mathrm{OE}_{-}$ exo. Secondly, miR-214 ${ }^{\mathrm{OE}}$-Exo are non-targeting agents which can also be absorbed by other cells. New targeted delivery methods, such as genetical or chemical modification of exosome's membrane, should be explored to avoid off-target effects. Thirdly, further studies are required to validate safety and efficacy of miR-214 ${ }^{\mathrm{OE}}$-Exo before it becomes a promising cell-free therapy for heart repair in clinical practice.

In conclusion, our study verified that miR-214 engineered huMSCs derived exosomes protected heart from ischemia injury through enhancing angiogenesis, promoting cell proliferation, inhibiting apoptosis, and reducing fibrosis in vivo and in vitro. PTEN/AKT signal pathway was one of the potential mechanisms involved in the biological activities of miR-214 ${ }^{\mathrm{OE}_{-}}$Exo. These findings suggest that the combination of MSC-derived exosomes and miRNAs may be a probable approach for the treatment of cardiovascular disease. 


\section{Declarations}

\section{Ethics approval and consent to participate}

Animal experiments were conducted according to the Guidelines for the Care and Use of Laboratory Animals and were approved by Ethics Committee of Xuzhou Central Hospital.

\section{Consent for publication}

Not applicable.

\section{Availability of data and materials}

Most of the datasets supporting the conclusions of this article are included within this article and the additional files. The datasets used or analyzed during the current study are available on reasonable request.

\section{Competing interests}

The authors have declared that no competing interest exists.

\section{Funding}

This study was supported by grants from the Major Research Plan of Changzhou Health Commission (ZD202020) and Young Talent Development Plan of Changzhou Health Commission (CZQM2020060).

\section{Authors' contributions}

WWZ, QJW, JZ, YJ, BH contributed to the design of the study. WWZ, QJW, JZ, LS, WD and YJW performed the experiments. WWZ, QJW, JZ, LS, WD, YJW, YJ and BH contributed to the writing the manuscript. WWZ, QJW and JZ contributed to the material support of the study. All authors read and approved the final manuscript.

\section{Acknowledgements}

Not applicable.

\section{References}

1. Zhou M, Wang H, Zeng X, et al. Mortality, morbidity, and risk factors in China and its provinces, 19902017: a systematic analysis for the Global Burden of Disease Study 2017. Lancet (London, England). 2019;394(10204):1145-1158.

2. Galiuto L, DeMaria A, Iliceto S. Microvascular damage during myocardial ischemia-reperfusion: pathophysiology, clinical implications and potential therapeutic approach evaluated by myocardial contrast echocardiography. Italian heart journal : official journal of the Italian Federation of Cardiology. 2000;1(2):108-116. 
3. Hou Z, Qin X, Hu Y, et al. Longterm Exercise-Derived Exosomal miR-342-5p: A Novel Exerkine for Cardioprotection. Circulation research. 2019;124(9):1386-1400.

4. Hinkel R, Ramanujam D, Kaczmarek V, et al. AntimiR-21 Prevents Myocardial Dysfunction in a Pig Model of Ischemia/Reperfusion Injury. Journal of the American College of Cardiology. 2020;75(15):1788-1800.

5. Gupta S, Foinquinos A, Thum S, et al. Preclinical Development of a MicroRNA-Based Therapy for Elderly Patients With Myocardial Infarction. Journal of the American College of Cardiology. 2016;68(14):1557-1571.

6. Lim G. MicroRNA-directed cardiac repair after myocardial infarction in pigs. Nature reviews Cardiology. 2019;16(8):454-455.

7. Rogg E, Abplanalp W, Bischof C, et al. Analysis of Cell Type-Specific Effects of MicroRNA-92a Provides Novel Insights Into Target Regulation and Mechanism of Action. Circulation. 2018;138(22):2545-2558.

8. Boon R, Dimmeler S. MicroRNAs in myocardial infarction. Nature reviews Cardiology. 2015;12(3):135142.

9. Zhu W, Sun L, Zhao P, et al. Macrophage migration inhibitory factor facilitates the therapeutic efficacy of mesenchymal stem cells derived exosomes in acute myocardial infarction through upregulating miR-133a-3p. Journal of nanobiotechnology. 2021;19(1):61.

10. Sun L, Zhu W, Zhao P, et al. Long noncoding RNA UCA1 from hypoxia-conditioned hMSC-derived exosomes: a novel molecular target for cardioprotection through miR-873-5p/XIAP axis. Cell death \& disease. 2020;11(8):696.

11. Sun L, Zhu W, Zhao P, et al. Down-Regulated Exosomal MicroRNA-221 - 3p Derived From Senescent Mesenchymal Stem Cells Impairs Heart Repair. Frontiers in cell and developmental biology. 2020;8:263.

12. Khatri N, Rathi M, Baradia D, Trehan S, Misra A. In vivo delivery aspects of miRNA, shRNA and siRNA. Critical reviews in therapeutic drug carrier systems. 2012;29(6):487-527.

13. Exosomes. Nature biotechnology. 2020;38(10):1150.

14. Hirai K, Ousaka D, Fukushima Y, et al. Cardiosphere-derived exosomal microRNAs for myocardial repair in pediatric dilated cardiomyopathy. Science translational medicine. 2020;12(573).

15. Ji J, Chen R, Zhao L, et al. Circulating exosomal mRNA profiling identifies novel signatures for the detection of prostate cancer. Molecular cancer. 2021;20(1):58.

16. Rodrigues G, Hoshino A, Kenific C, et al. Tumour exosomal CEMIP protein promotes cancer cell colonization in brain metastasis. Nature cell biology. 2019;21(11):1403-1412.

17. Chen Y, Tian Z, He L, et al. Exosomes derived from miR-26a-modified MSCs promote axonal regeneration via the PTEN/AKT/mTOR pathway following spinal cord injury. Stem cell research \& therapy. 2021;12(1):224. 
18. Huang J, Xu Y, Yin X, Lin F. Exosomes Derived from miR-126-modified MSCs Promote Angiogenesis and Neurogenesis and Attenuate Apoptosis after Spinal Cord Injury in Rats. Neuroscience. 2020;424:133-145.

19. Cho $Y$, Cha M, Song B, et al. Enhancement of MSC adhesion and therapeutic efficiency in ischemic heart using lentivirus delivery with periostin. Biomaterials. 2012;33(5):1376-1385.

20. Jiang X, Yang Z, Dong M. Cardiac repair in a murine model of myocardial infarction with human induced pluripotent stem cell-derived cardiomyocytes. Stem cell research \& therapy. 2020;11(1):297.

21. Wysoczynski M, Bolli R. A realistic appraisal of the use of embryonic stem cell-based therapies for cardiac repair. European heart journal. 2020;41(25):2397-2404.

22. Lv G, Shao S, Dong H, Bian X, Yang X, Dong S. MicroRNA-214 protects cardiac myocytes against H2O2-induced injury. Journal of cellular biochemistry. 2014;115(1):93-101.

23. van Balkom $B$, de Jong 0, Smits $M$, et al. Endothelial cells require miR-214 to secrete exosomes that suppress senescence and induce angiogenesis in human and mouse endothelial cells. Blood. 2013;121(19):3997-4006, S3991-3915.

24. Zhang Y, Zhu W, He H, et al. Macrophage migration inhibitory factor rejuvenates aged human mesenchymal stem cells and improves myocardial repair. Aging. 2019;11(24):12641-12660.

25. Gandhi N, Tekade R, Chougule M. Nanocarrier mediated delivery of siRNA/miRNA in combination with chemotherapeutic agents for cancer therapy: current progress and advances. Journal of controlled release : official journal of the Controlled Release Society. 2014;194:238-256.

26. Marshall E. Gene therapy death prompts review of adenovirus vector. Science (New York, NY). 1999;286(5448):2244-2245.

27. Kamps J, Krenning G. Micromanaging cardiac regeneration: Targeted delivery of microRNAs for cardiac repair and regeneration. World journal of cardiology. 2016;8(2):163-179.

28. O'Loughlin A, Mäger I, de Jong 0 , et al. Functional Delivery of Lipid-Conjugated siRNA by Extracellular Vesicles. Molecular therapy : the journal of the American Society of Gene Therapy. 2017;25(7):15801587.

29. Cully M. Exosome-based candidates move into the clinic. Nature reviews Drug discovery. 2021;20(1):6-7.

30. O'Brien K, Breyne K, Ughetto S, Laurent L, Breakefield X. RNA delivery by extracellular vesicles in mammalian cells and its applications. Nature reviews Molecular cell biology. 2020;21(10):585-606.

31. Janas T, Janas P, Sapoń K, Janas T. Binding of RNA Aptamers to Membrane Lipid Rafts: Implications for Exosomal miRNAs Transfer from Cancer to Immune Cells. International journal of molecular sciences. 2020;21(22).

32. Sung B, von Lersner A, Guerrero J, et al. A live cell reporter of exosome secretion and uptake reveals pathfinding behavior of migrating cells. Nature communications. 2020;11(1):2092.

33. Mayourian J, Ceholski D, Gorski P, et al. Exosomal microRNA-21-5p Mediates Mesenchymal Stem Cell Paracrine Effects on Human Cardiac Tissue Contractility. Circulation research. 2018;122(7):933-944. 
34. Liu X, Li X, Zhu W, et al. Exosomes from mesenchymal stem cells overexpressing MIF enhance myocardial repair. Journal of cellular physiology. 2020;235(11):8010-8022.

35. Zhuang L, Xia W, Chen D, et al. Exosomal LncRNA-NEAT1 derived from MIF-treated mesenchymal stem cells protected against doxorubicin-induced cardiac senescence through sponging miR-221-3p. Journal of nanobiotechnology. 2020;18(1):157.

36. Luo Q, Guo D, Liu G, Chen G, Hang M, Jin M. Exosomes from MiR-126-Overexpressing Adscs Are Therapeutic in Relieving Acute Myocardial Ischaemic Injury. Cellular physiology and biochemistry: international journal of experimental cellular physiology, biochemistry, and pharmacology. 2017;44(6):2105-2116.

37. Ma J, Zhao Y, Sun L, et al. Exosomes Derived from Akt-Modified Human Umbilical Cord Mesenchymal Stem Cells Improve Cardiac Regeneration and Promote Angiogenesis via Activating Platelet-Derived Growth Factor D. Stem cells translational medicine. 2017;6(1):51-59.

38. Aurora A, Mahmoud A, Luo X, et al. MicroRNA-214 protects the mouse heart from ischemic injury by controlling $\mathrm{Ca}^{2} \rrbracket$ overload and cell death. The Journal of clinical investigation. 2012;122(4):12221232.

39. Yang X, Qin Y, Shao S, et al. MicroRNA-214 Inhibits Left Ventricular Remodeling in an Acute Myocardial Infarction Rat Model by Suppressing Cellular Apoptosis via the Phosphatase and Tensin Homolog (PTEN). International heart journal. 2016;57(2):247-250.

40. Wang Y, Zhao R, Liu D, et al. Exosomes Derived from miR-214-Enriched Bone Marrow-Derived Mesenchymal Stem Cells Regulate Oxidative Damage in Cardiac Stem Cells by Targeting CaMKII. Oxidative medicine and cellular longevity. 2018;2018:4971261.

\section{Figures}


A

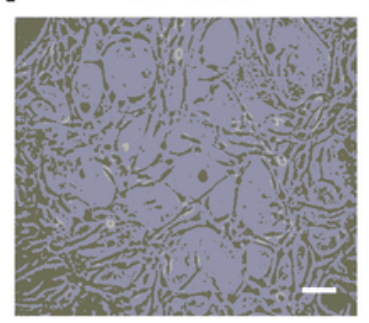

B Adipogenesis

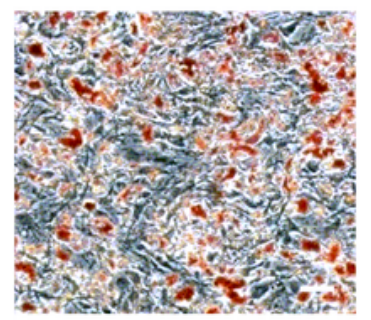

D

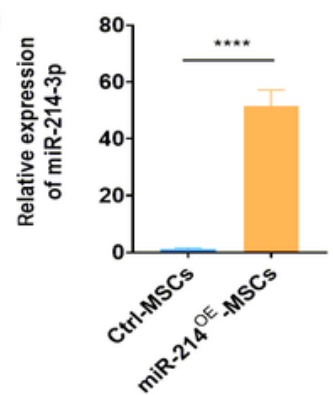

F Ctrl-Exo

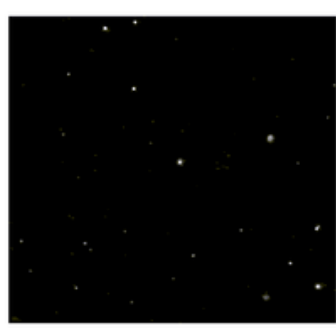

H

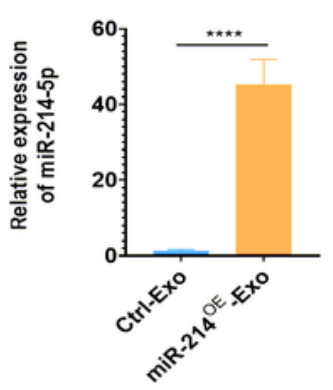

miR-214 ${ }^{\text {OE-EXO }}$
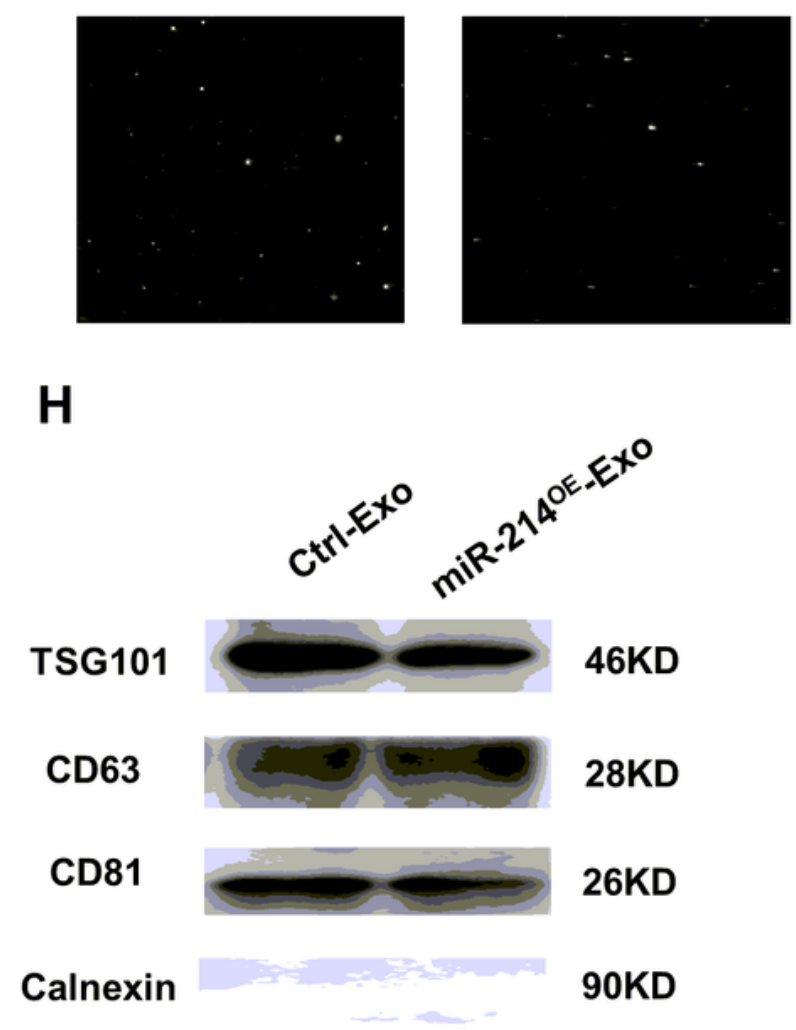

Chondrogenesis C Ctrl-MSCs

miR-214 ${ }^{\text {OE-MSCs }}$
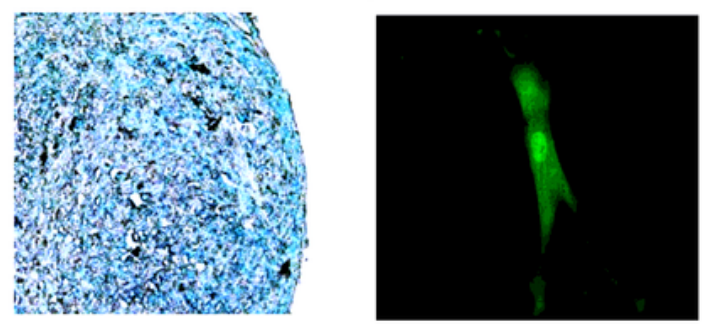

E

Ctrl-Exo

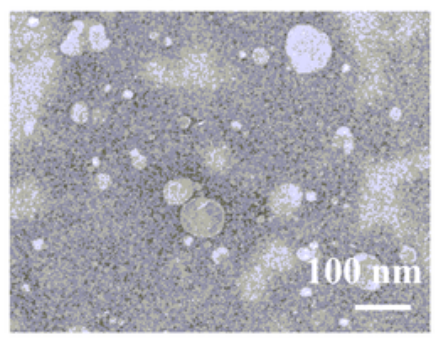

G

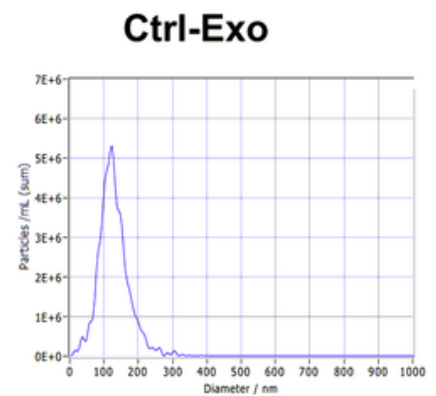

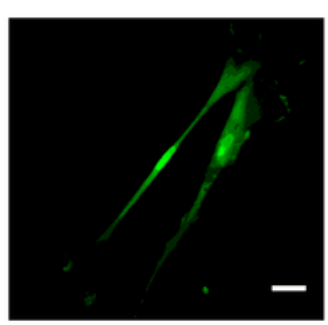

miR-214 ${ }^{\mathrm{OE}_{\text {-ExO }}}$

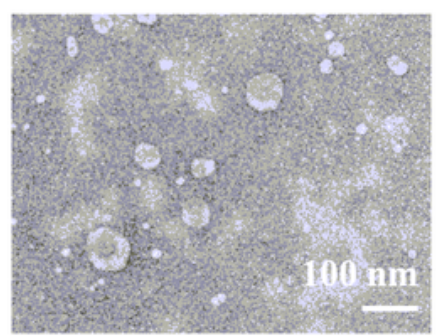

miR-214 ${ }^{O E}$-Exo

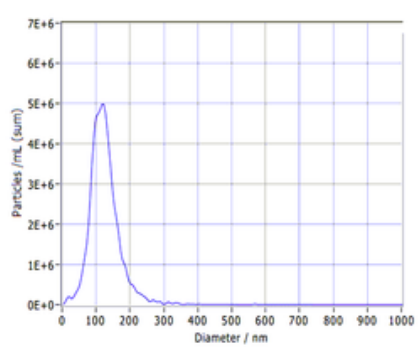

HUVEC

(

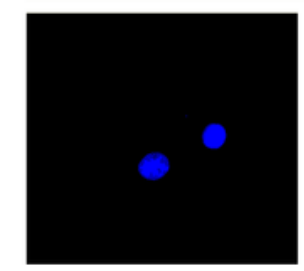

Control

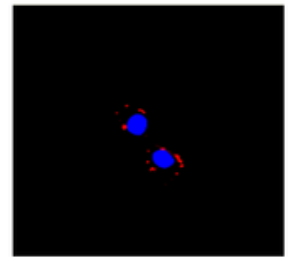

$6 \mathrm{~h}$

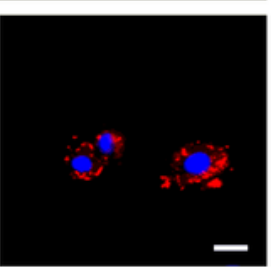

$24 \mathrm{~h}$

NRCM

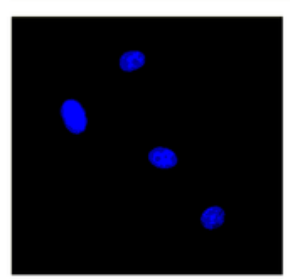

Control

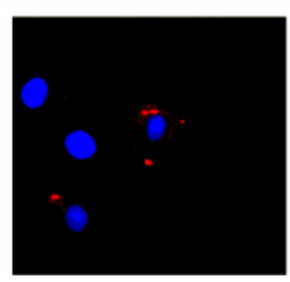

$6 \mathrm{~h}$

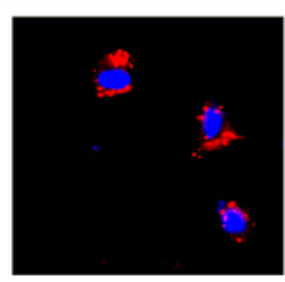

$24 \mathrm{~h}$

Figure 1

Characterization of huMSCs and exosomes which are derived from huMSCs. (A-B) Morphology and multiple differentiation potential of huMSCs. Scale bar $=100 \mu \mathrm{m}$. (C) Fluorescence microscopy image of lentivirus transfection in Ctrl-MSC and miR-2140E-MSCs groups. Scale bar $=20 \mu \mathrm{m}$. (D) The expression of miR-214-3p in cells and exosomes was verified by real-time PCR. (E) Cup-shaped morphology of purified Ctrl-Exo and miR-2140E- Exo assessed by TEM. (F) NTA representative screen shot video; the bright white 
point represents a particle movement. (G) The particle size distribution and particle concentration were analyzed by nanoparticle tracking analysis. $(H)$ The exosomal protein markers of TSG101, CD63, and CD81 in Ctrl-Exo and miR-2140E-Exo groups. (I) Confocal images of Dil labeled exosomes (red fluorescence) were endocytosed by HUVECs and NRCMs 6 and $24 \mathrm{~h}$ after incubation. Scale bar $=20 \mu \mathrm{m}$.

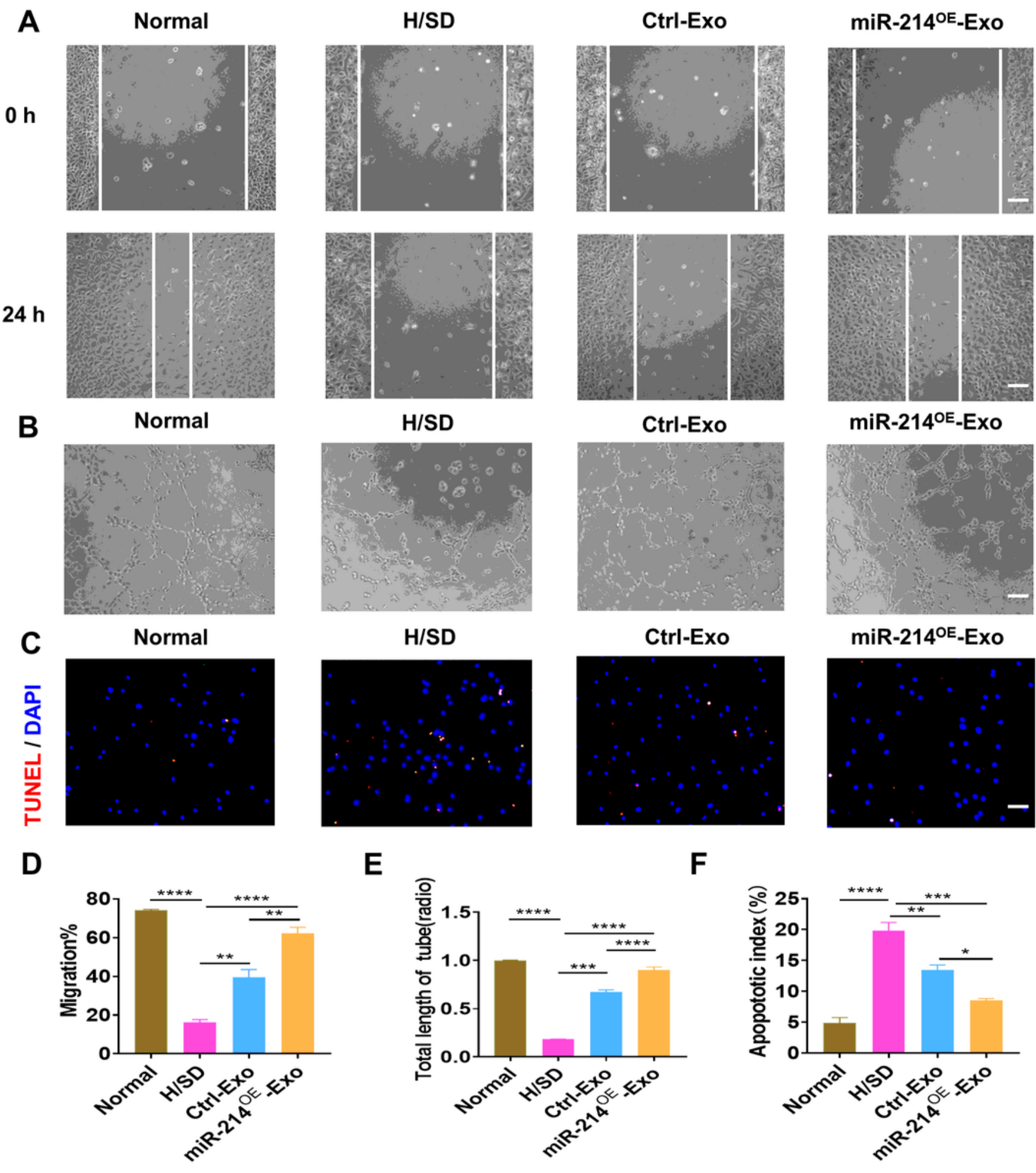

Figure 2 
Effects of miR-2140E-Exo on HUVECs and NRCMs. (A, D) Migration of HUVECs cultured with PBS, CtrlExo and miR-2140E-Exo was showed in Figure A \& D. Scale bar $=100 \mu \mathrm{m}$. (B, E) Tube formation of HUVECs incubated with PBS, Ctrl-Exo and miR-2140E-Exo and quantification analysis. Scale bar $=100$ $\mu \mathrm{m}$. (C, F) Representative images of the apoptosis determined by TUNEL staining (red) in NRCMs among different groups and Quantitative analysis of the percentage of the apoptotic NRCMs among the different groups. Scale bar $=50 \mu \mathrm{m}$. Continuous and categorical variables were described by means \pm SEM and percentages, respectively. $\mathrm{n}=3$ for each group. ${ }^{*} \mathrm{P}<0.05$; ${ }^{* * P}<0.01 ; * \star * P<0.001$, $* \star * * P<0.0001$.

A

Experimental flowchart for in vivo study

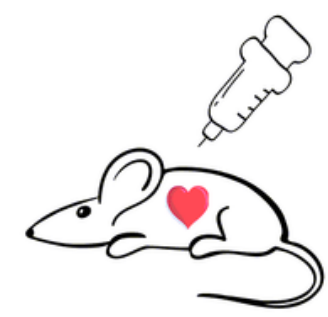

B

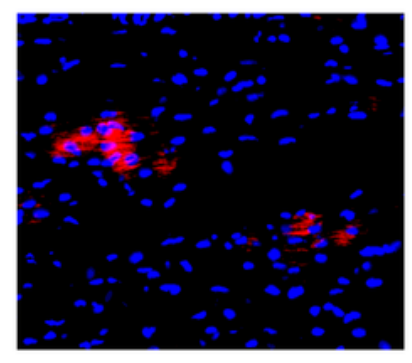

C

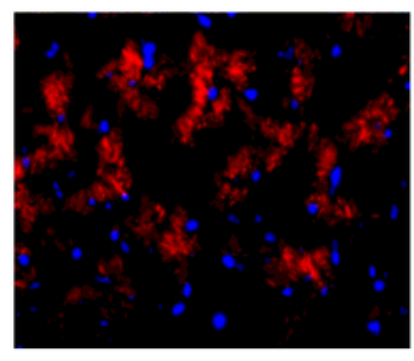

D

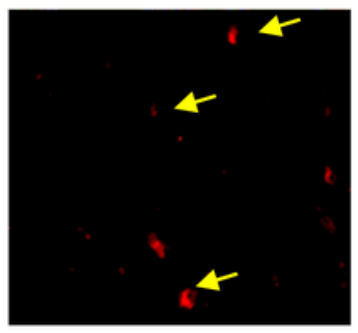

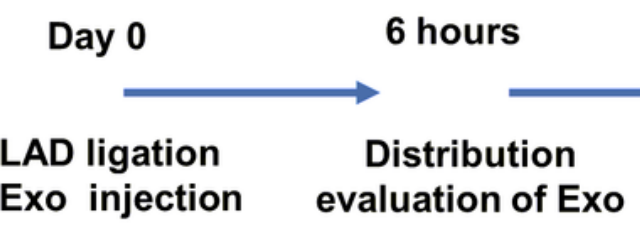

Dil-Exosome/CD31/Dapi
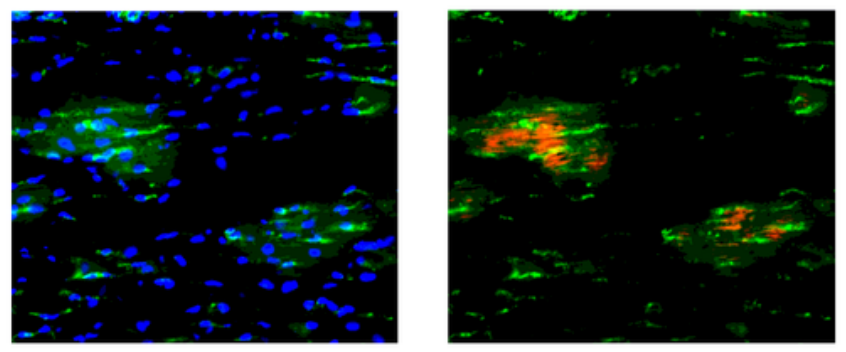

Dil-Exosome/a-actin/Dapi
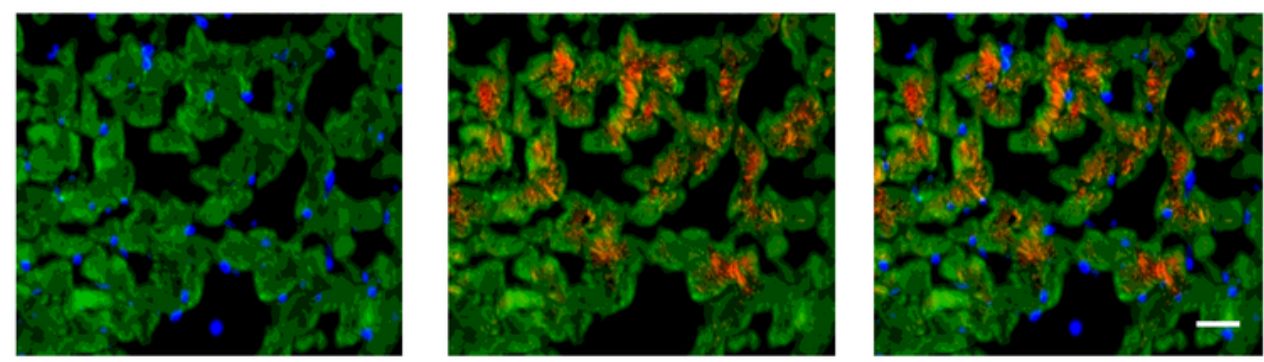

4 weeks

Echocardiography

Echocardiography HE\& Masson staining Capillaries \& Arterioles TUNEL

Cl-caspase3+

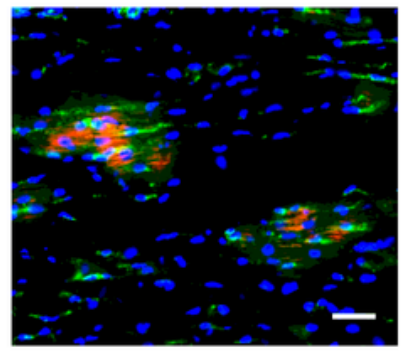

Cy3-miR-214 probe/a-actin/Dapi
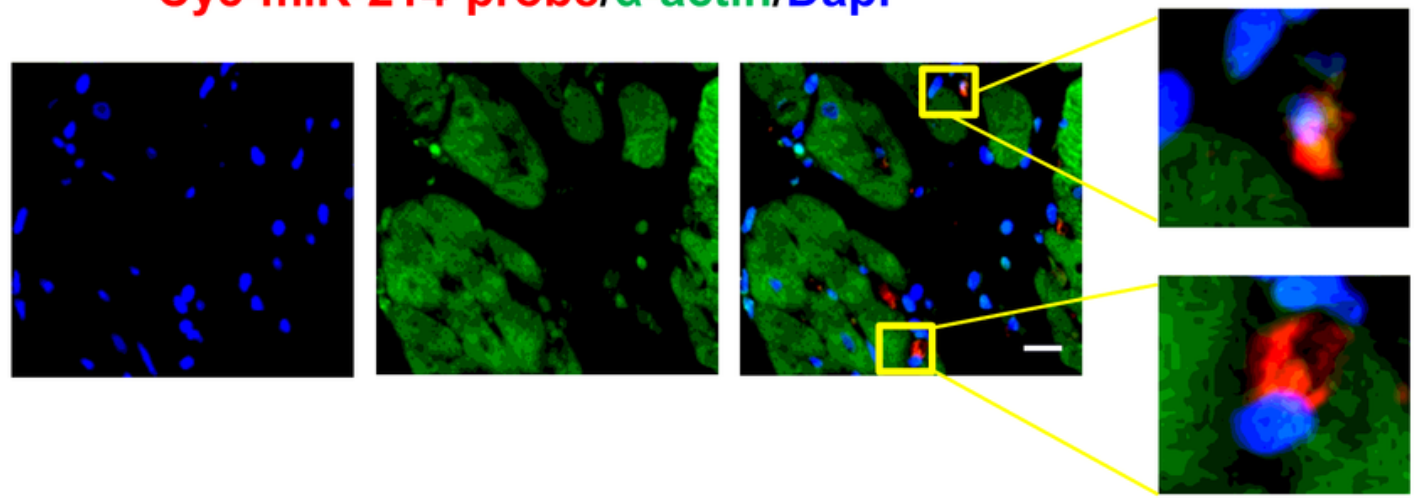
Figure 3

Distribution of miR-214-loaded exosomes in infarcted hearts. (A) Flow chart of in vivo experiment design. (B-C) The expression level of CD31 (B) and a-actin (C) protein was detected by immunofluorescence $6 \mathrm{~h}$ after intramyocardial injection of Dil labeled exosomes. Scale bar $=50 \mu \mathrm{m}$. (D) FISH analysis in infarcted LV myocardium of SD Rats. Cy3-miR-214 probe (red) was used to detect miR-214. Concentrated miR-214 was indicated by yellow arrowheads. Scale bar $=20 \mu \mathrm{m}$.
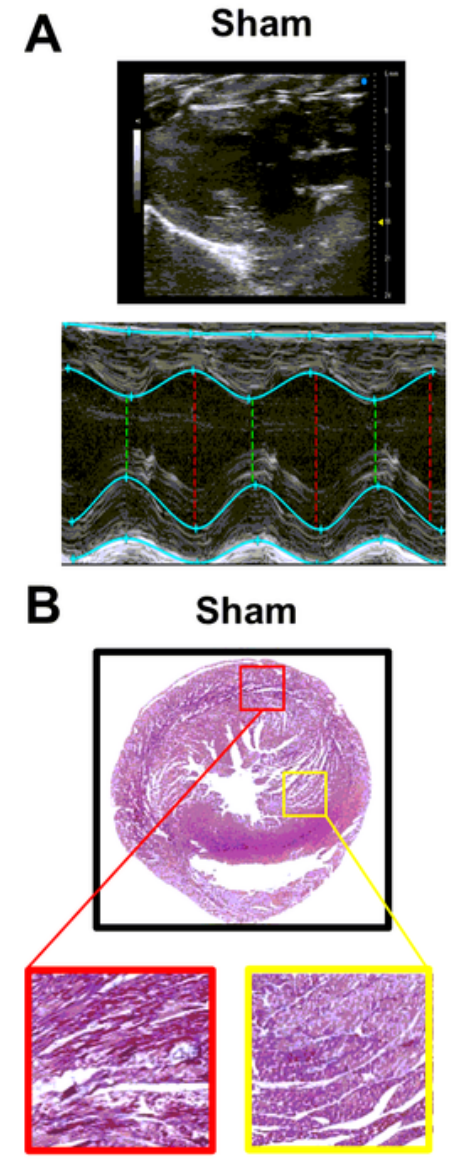

C
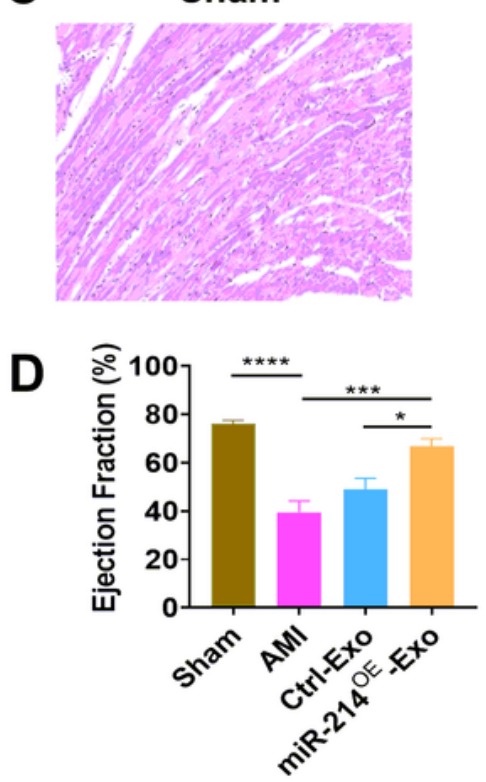

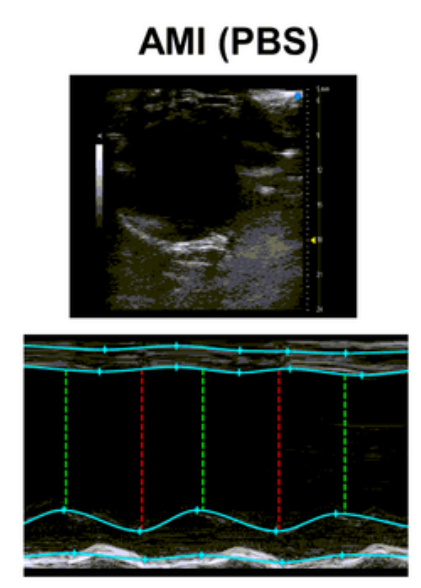

AMI (PBS)

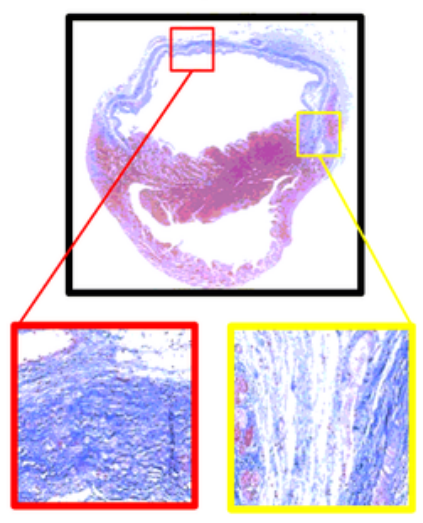

AMI (PBS)
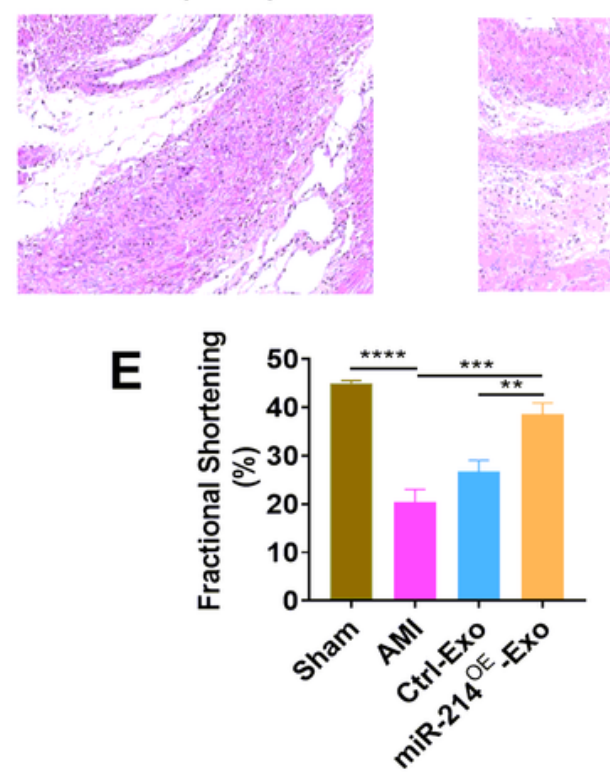

Ctrl-Exo

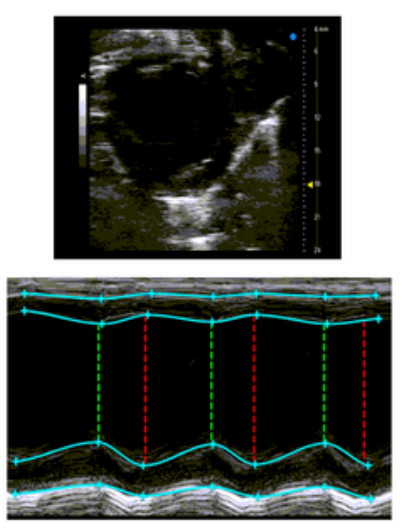

Ctrl-Exo

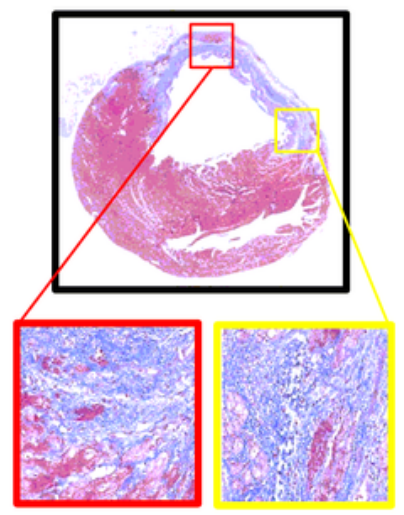

Ctrl-Exo

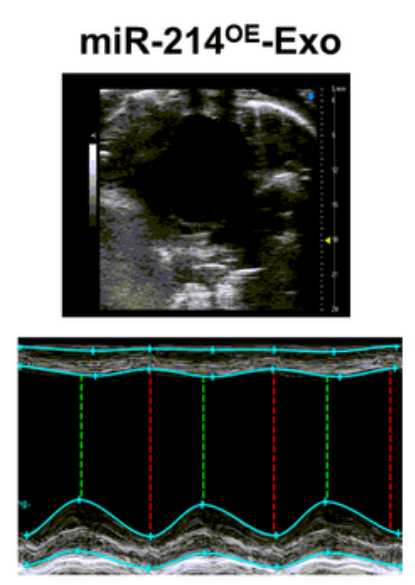

miR-214 ${ }^{\text {OE-ExO }}$

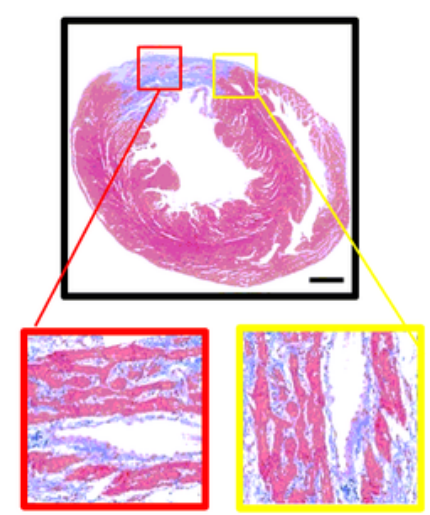

miR-214 ${ }^{\mathrm{OE}}$-ExO
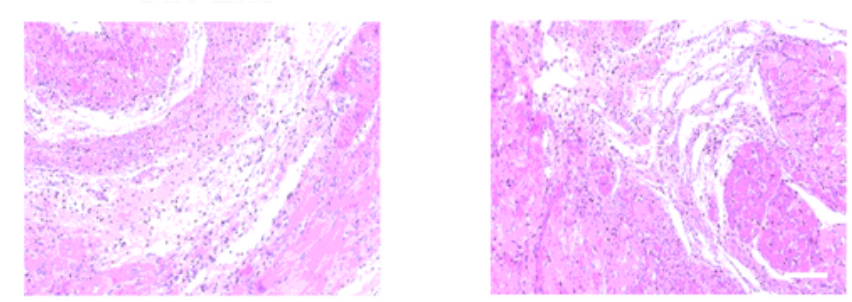

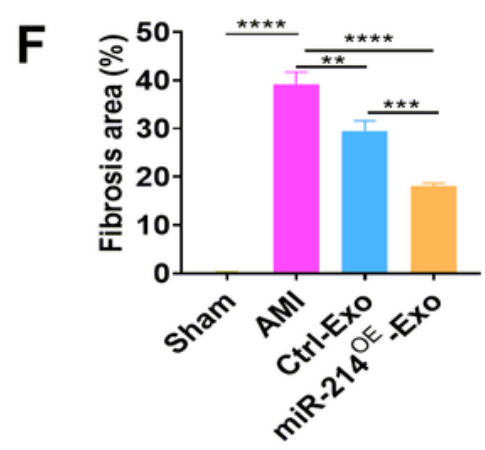




\section{Figure 4}

miR-2140E-Exo effectively preserved cardiac function in rats with $\mathrm{MI}$ in vivo. (A) Representative images of M-mode echocardiography captured 28 days after MI between different groups. (B) The representative Masson's trichrome-stained myocardial cross-sections 4 weeks after MI with the injection of PBS, Ctrl-Exo, and miR-2140E-Exo. Red frame indicates the infarct zone and yellow frame indicates the border zone. Scale bar $=20 \mu \mathrm{m}$. (C) HE staining at the border zone 28 days post MI. (D-E) Left ventricular ejection fraction (LVEF) and left ventricular fractional shortening (LVFS) measured by echocardiography 28 days after MI. (F) Quantitative analysis of heart fibrosis between different groups. 

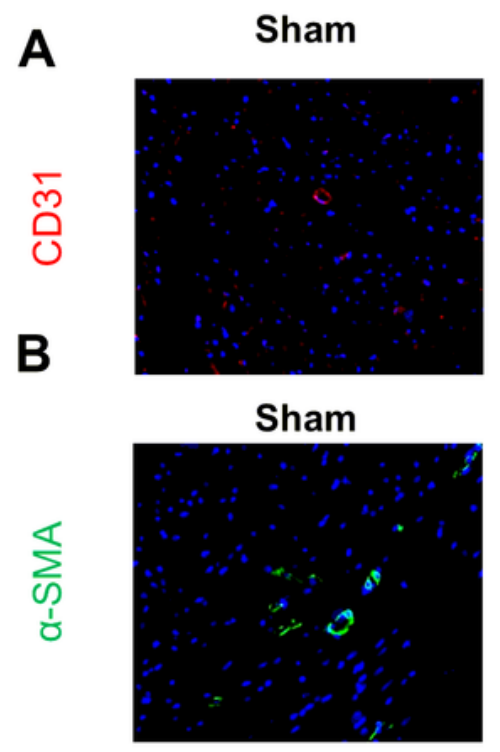

C

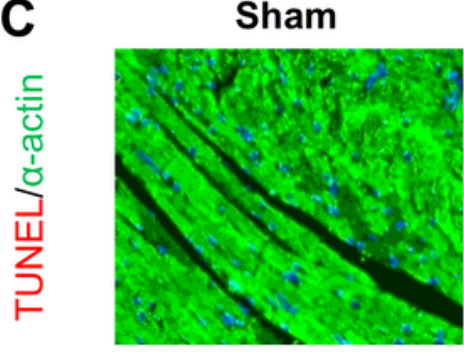

D
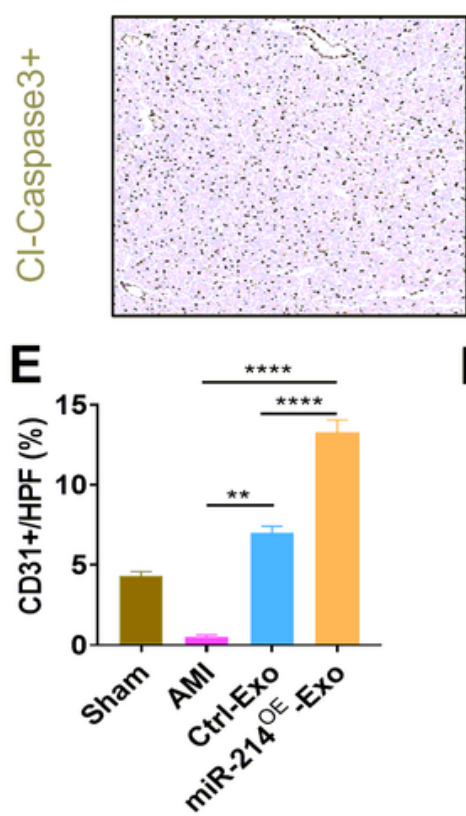

AMI (PBS)

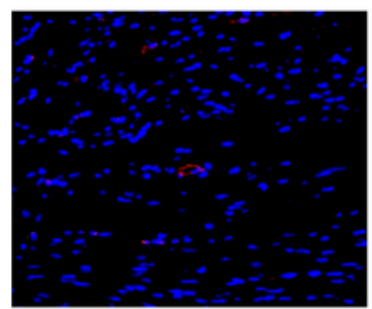

AMI (PBS)

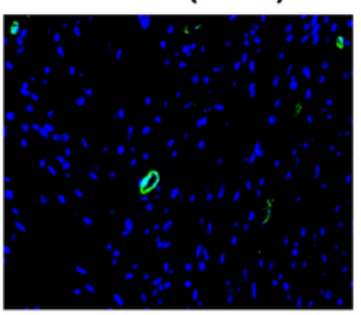

AMI (PBS)

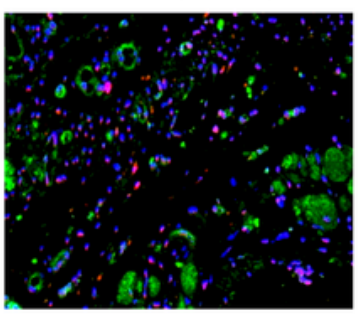

AMI (PBS)

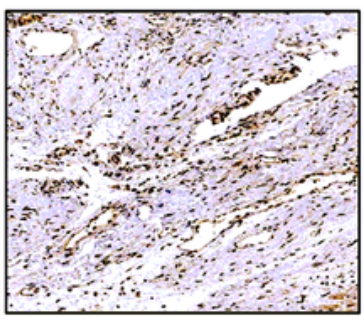

$\mathbf{F}$

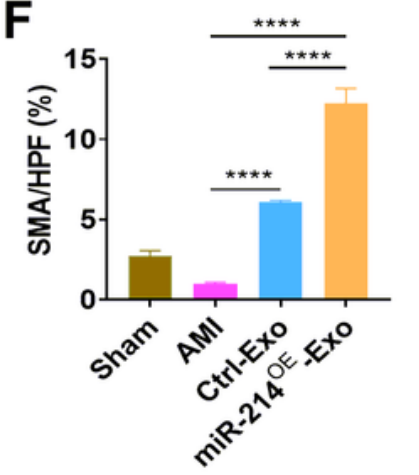

Ctrl-Exo

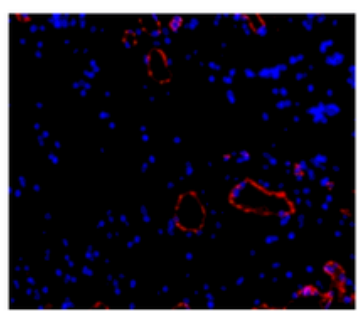

Ctrl-Exo

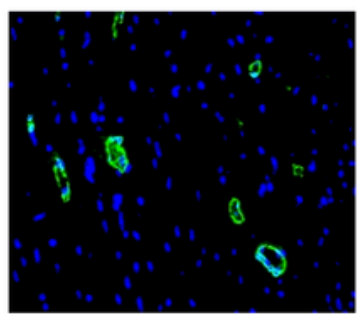

Ctrl-Exo

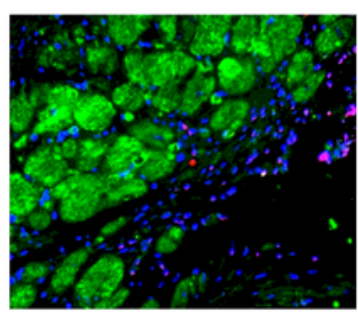

Ctrl-Exo

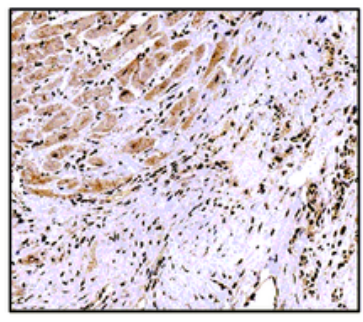

G

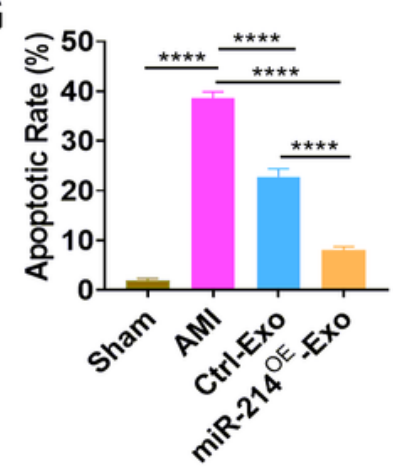

miR-214 ${ }^{O E}$-ExO

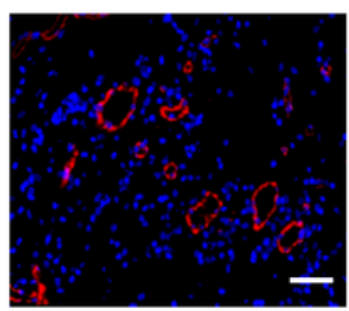

miR-214 ${ }^{\mathrm{OE}}$-ExO

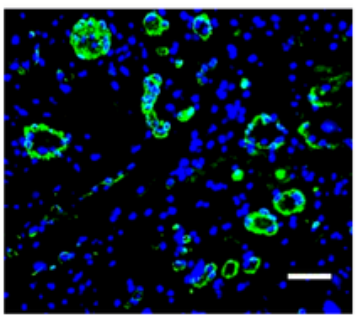

MiR-214 ${ }^{\text {OE }}$-Exo

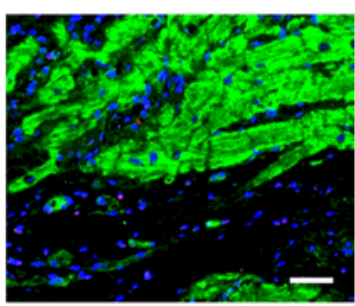

MiR-214 ${ }^{O E}$-Exo

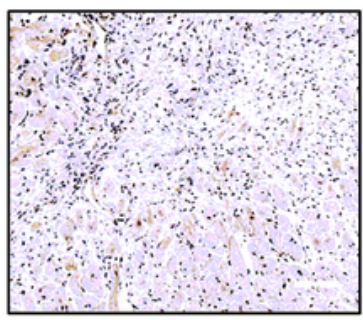

H

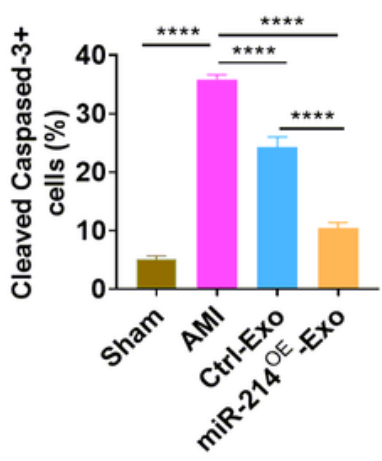

Figure 5

miR-2140E-Exo promoted angiogenesis and cardiomyocyte survival in infarcted hearts. (A) Neovascularization at the border zone 28 days after MI was evaluated by staining with CD31 (red) and nuclei (blue). Scale bar= $50 \mu \mathrm{m}$. (B) a-SMA positively stained arterioles at the border zone 4 weeks after MI. Scale bar $=50 \mu \mathrm{m}$. (C) Representative images showing the terminal deoxynucleotidyl transferasemediated dUTP nick end-labeling (TUNEL) positive cells in the heart tissue among the different groups. 
Scale bar $=50 \mu \mathrm{m}$. (D) Cleaved Caspase-3 staining at the border zone 28 days post MI. Scale bar= $50 \mu \mathrm{m}$. ( $E$ and F) Quantitative analysis of the CD31(E) and a-SMA(F) density in the heart tissue of rats from the different groups. (G) Quantitative analysis of the apoptotic rates among the different groups. $(H)$ Quantitative analysis of cleaved-caspase-3+ cells at the border zone among the different groups.
A
H/SD
B
Ctrl-Exo

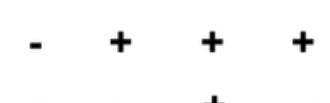
H/SD +++
miR-2140E-EXO - ++
miR-214 ${ }^{O E}$-Exo
MK2206
GAPDH

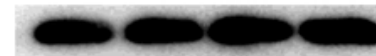
37KD
GAPDH
37KD
AKT

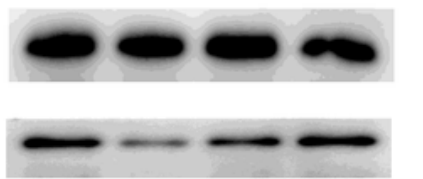
60KD
AKT

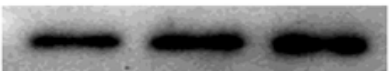
60KD
p-AKT
60KD
p-AKT

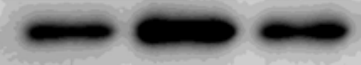
60KD
$\mathrm{Bcl} 2$

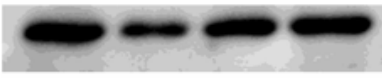
26KD
$\mathrm{Bcl} 2$

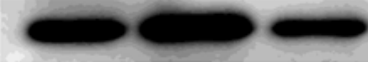
26KD
Bax
21KD
Bax
21KD

C

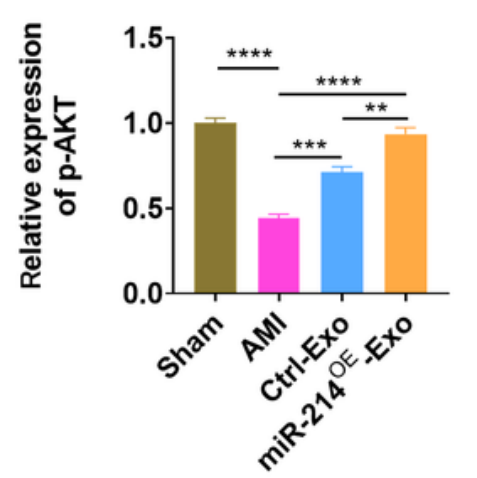

E
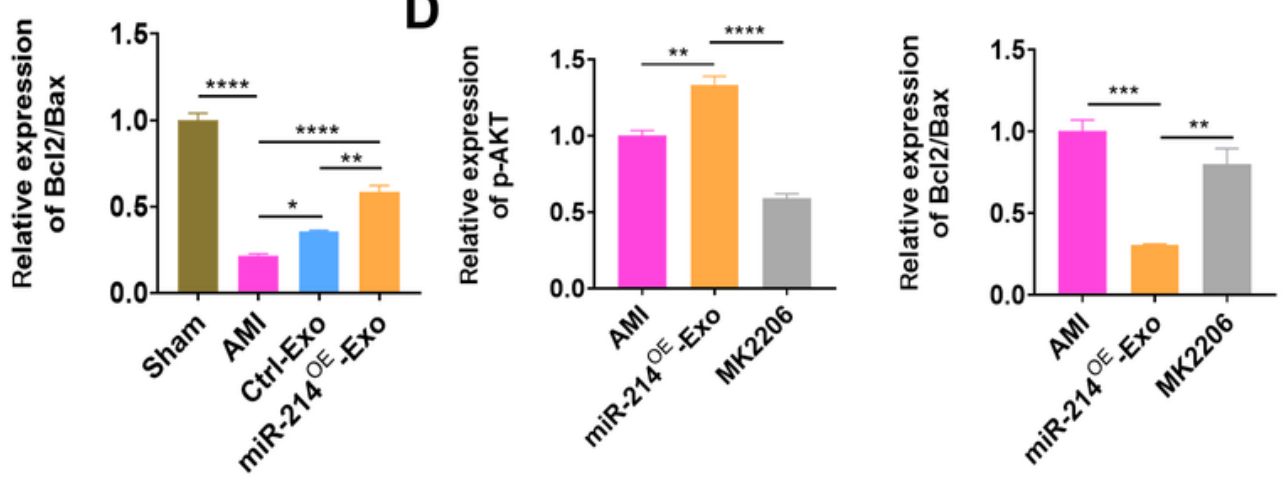

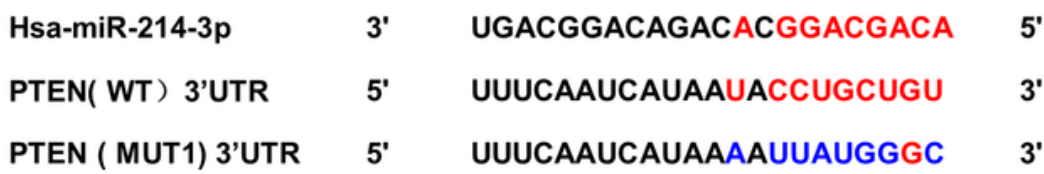

F

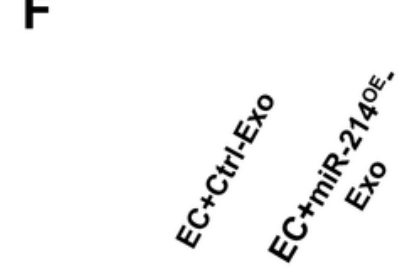

GAPDH

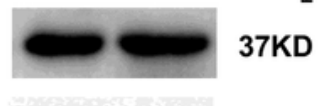

PTEN

21KD

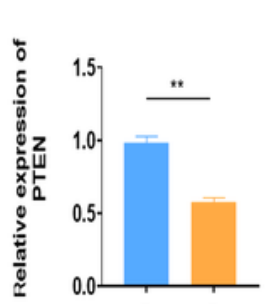

G

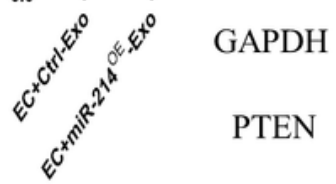

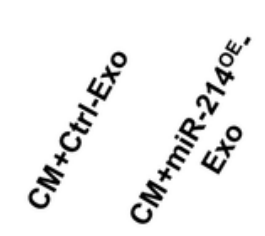

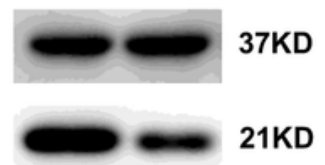

H

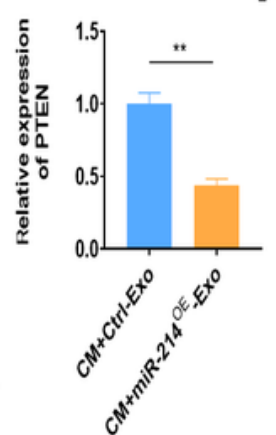

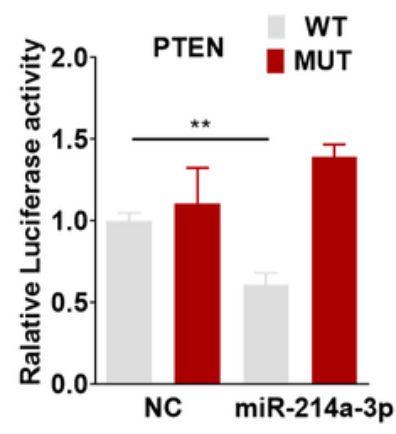

Figure 6 
Exosomal miR-214-3p attenuated cardiomyocyte injury via regulation of $p$-AKT by targeting PTEN (A)

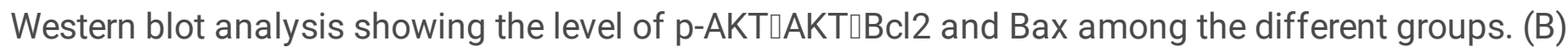
Neonatal rat cardiomyocytes (NRCMs) were treated with miR-2140E-Exo or miR-2140E-Exo+MK2206,

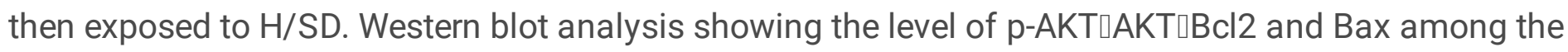

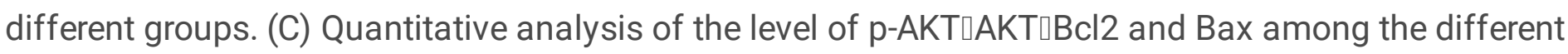
groups. (D) Quantitative analysis of the level of p-AKT\AKT\Bcl2 and Bax in figure 6B among the different groups. (E) The predicted miR-214 targeting sequence in the 3'-UTR of PTEN. (F) Western blot analysis showing the PTEN levels after administration of Ctrl-Exo or miR-2140E-Exo in HUVECs. (G) Western blot analysis of PTEN after administration of Ctrl-Exo or miR-2140E-Exo in NRCMs. (H) Luciferase reporter assay was performed to confirm the target gene of miR-214. 
A B $\quad C_{\text {miR-214 }}{ }^{\text {EE-Exo+Vector miR-214 }}{ }_{\text {OE-EXo+PTEN }}$ D
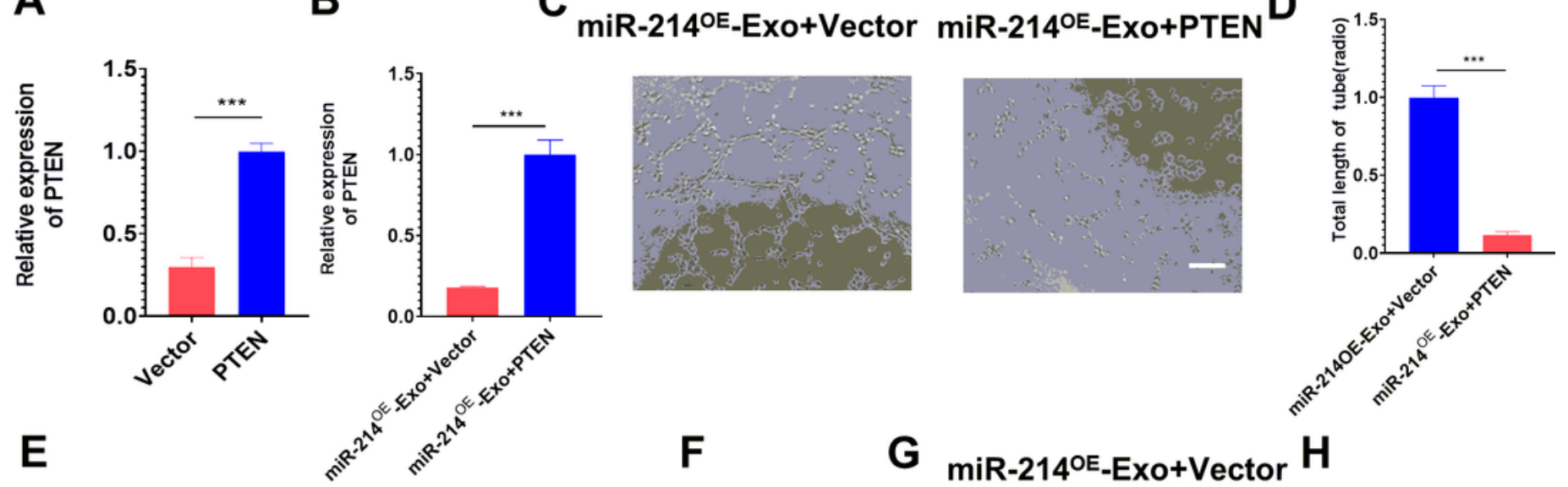

miR-214OE-Exo+Vector miR-214 ${ }^{\mathrm{OE}}$-Exo+PTEN

$\mathbf{O h}$
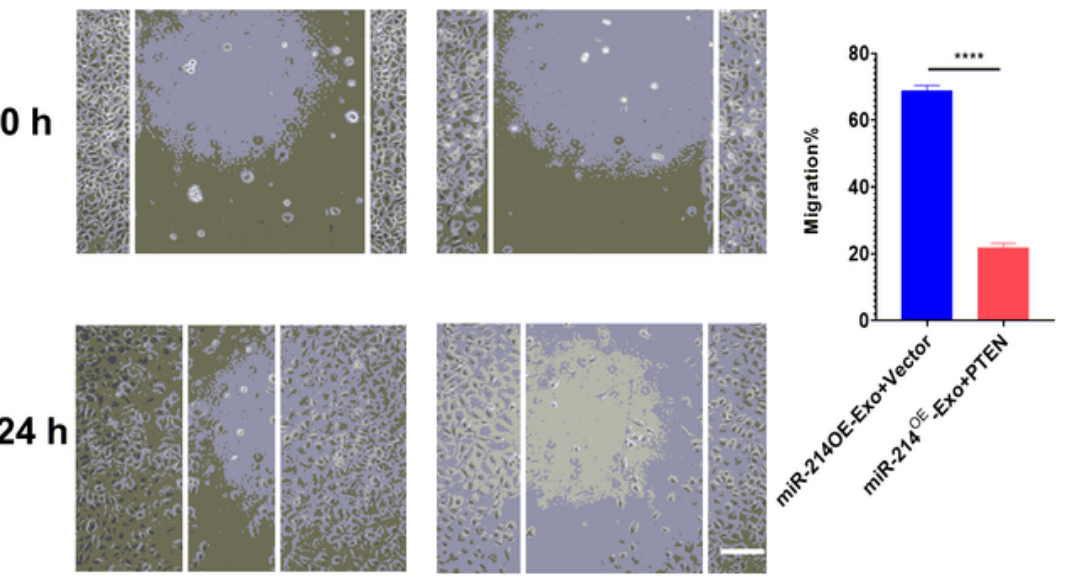

G miR-214 ${ }^{\text {OE }}$-Exo+Vector $\mathrm{H}$

$\mathbf{F}$

I
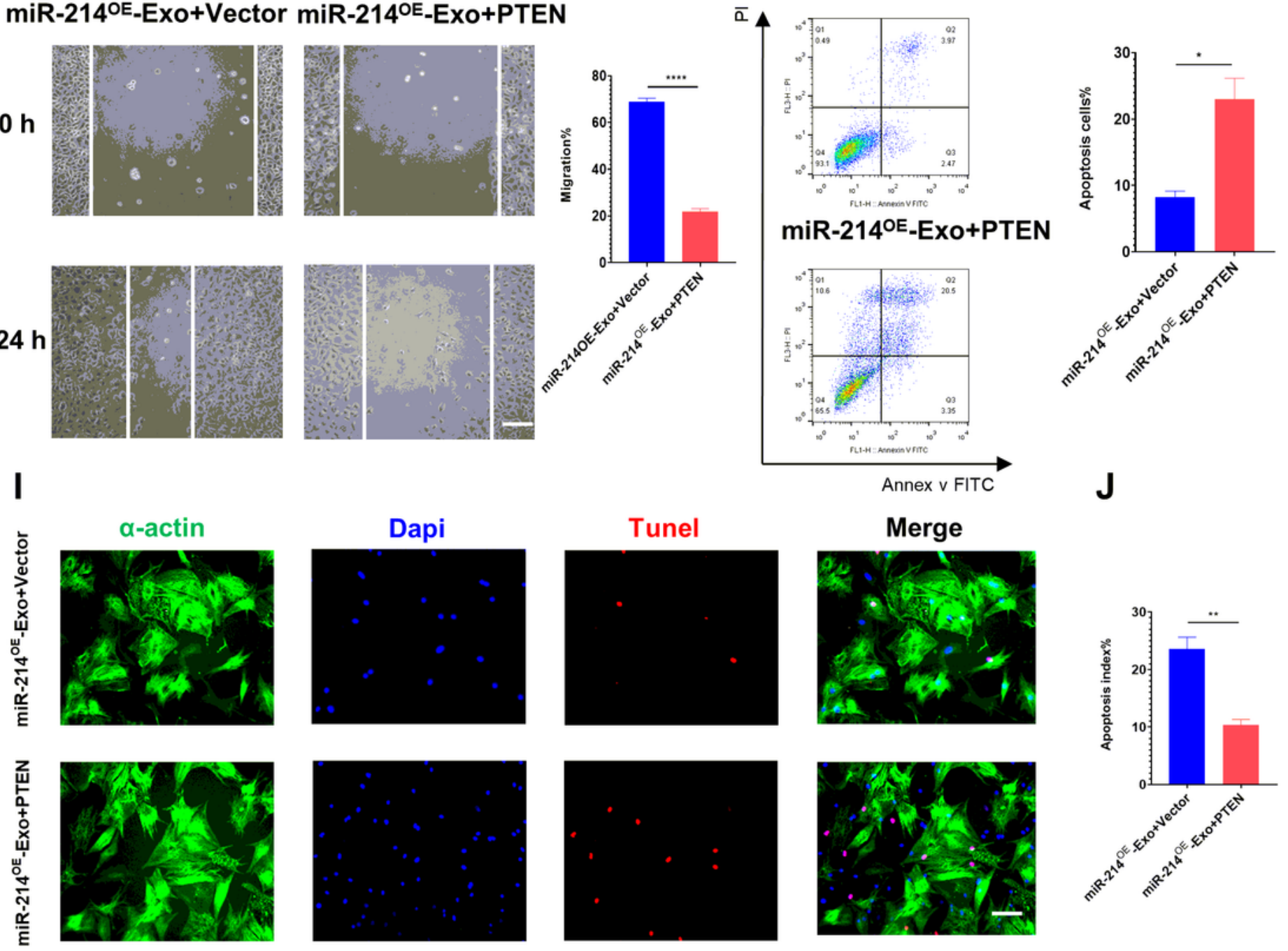

Figure 7

miR-2140E-Exo promote migration, tube formation and apoptosis inhibition by targeting PTEN (A) Expression level of PTEN in HUVECs after transfection with plasmids containing Vector or PTEN. (B) Expression level of PTEN in HUVECs after transfection with Vector or PTEN followed by administration miR-2140E-Exo. (C-D) Tube formation assays were utilized to verify the functional role of PTEN on angiogenesis in HUVECs. Scale bar $=100 \mu \mathrm{m}$. (E-F) Scratch assays were used to validate the functional 
effect of PTEN on migration of HUVECs. Scale bar $=100 \mu \mathrm{m}$. (G-H) The apoptosis rate of HUVECs transfected with plasmids containing Vector or PTEN followed by administration miR-2140E-Exo was detected by Annexin V/FITC/PI double-staining flow cytometry. (I-J) The apoptosis rate of NRCMs transfected with plasmids containing Vector or PTEN followed by administration miR-2140E-Exo was detected by TUNEL staining (red). Scale bar $=50 \mu \mathrm{m}$.

\section{Supplementary Files}

This is a list of supplementary files associated with this preprint. Click to download.

- SUPP.pdf 